\title{
EXPONENTIAL UTILITY INDIFFERENCE VALUATION IN TWO BROWNIAN SETTINGS WITH STOCHASTIC CORRELATION
}

\author{
CHRISTOPH FREI ${ }^{* * *}$ AND \\ MARTIN SCHWEIZER, ${ }^{* * * *}$ ETH Zürich
}

\begin{abstract}
We study the exponential utility indifference valuation of a contingent claim $B$ in an incomplete market driven by two Brownian motions. The claim depends on a nontradable asset stochastically correlated with the traded asset available for hedging. We use martingale arguments to provide upper and lower bounds, in terms of bounds on the correlation, for the value $V^{B}$ of the exponential utility maximization problem with the claim $B$ as random endowment. This yields an explicit formula for the indifference value $b$ of $B$ at any time, even with a fairly general stochastic correlation. Earlier results with constant correlation are recovered and extended. The reason why all this works is that, after a transformation to the minimal martingale measure, the value $V^{B}$ enjoys a monotonicity property in the correlation between tradable and nontradable assets.
\end{abstract}

Keywords: Exponential utility; indifference valuation; nontradable asset; stochastic volatility; random endowment; distortion power; correlated Brownian motion

2000 Mathematics Subject Classification: Primary 91B28

Secondary 60G35; 93E20

\section{Introduction}

It is widely accepted that valuing contingent claims in incomplete markets needs subjective criteria in addition to absence of arbitrage. Utility indifference provides one such criterion, which has an economic justification because the chosen utility function reflects the individual investor's risk preferences. The basic idea of this approach is that the investor should achieve the same expected utility in the two cases where (i) he is free of obligation or (ii) he has to pay out the contingent claim $B$, but has an initial capital increased by the amount of the indifference (seller) value of $B$. A precise mathematical formulation leads to a pair of optimization problems over investment strategies. By using dual methods, which involve finding an optimal probability measure instead of an optimal strategy, one can derive some general properties for the exponential utility indifference value of a claim; see, for instance, [3] or [15], or [1] for an approach via backward stochastic differential equations in a model with jumps. However, it is difficult to derive a closed-form formula for the indifference value even in an explicit model.

Our basic model presented in Section 2 consists of a risk-free bank account and a stock $S$ driven by a Brownian motion $W$. The contingent claim $B$ to be valued depends on another

Received 26 June 2007; revision received 28 March 2008.

* Postal address: Department of Mathematics, ETH Zürich, 8092 Zürich, Switzerland.

** Email address: christoph.frei@math.ethz.ch

*** Email address: martin.schweizer@math.ethz.ch 
Brownian motion $Y$, which has stochastic instantaneous correlation $\rho$ with $W$. Indifference valuation is done via exponential utility. In the literature, which we compare in Section 4 with our results, there are two main approaches to obtain explicit formulae for the value of the resulting optimization problem. In a Markovian setting, Henderson [7], Henderson and Hobson [9], [10], and Musiela and Zariphopoulou [18], among others, started with the Hamilton-JacobiBellman nonlinear partial differential equation (PDE) for the value function of the underlying stochastic control problem. This PDE was then linearized by a power transformation with a constant exponent called the distortion power. This method works only if one has a Markovian model and if $\rho$ is constant. In an alternative approach, Tehranchi [21] first proved a Hölder-type inequality which he then applied to solve the portfolio optimization problem. The distortion power there arises as an exponent from the Hölder-type inequality. Tehranchi found an explicit expression for the indifference value at time 0 if $\rho$ is constant. While this method needs no Markovian assumption and can treat claims which are general (bounded) functionals of the process $Y$, it is still restricted to situations with constant correlation.

Since (exponential) utility indifference valuation hinges on (exponential) utility maximization with a random endowment, we start by tackling the latter. With the goal of deriving explicit results in our Brownian setting, in Subsection 2.2 we provide the motivation for the introduction of an auxiliary abstract optimization problem in a martingale framework. Our main theoretical result is Theorem 1 in Section 3; it gives an explicit formula for the value of this abstract problem. The proof uses martingale arguments to give upper and lower bounds on that value, in terms of bounds on $\rho$. Crucially, these bounds have the same structure, which enables us to derive a closed-form expression by interpolation. In particular, this allows us to handle a random correlation $\rho$.

Section 4 contains two applications of Theorem 1 . In the first, case (I), we extend the model of [21] to a fairly general stochastic correlation; the typical example is a model with stochastic volatility which is correlated with the stock in a nondeterministic way. In the second, case (II), the asset driving the claim $B$ is traded in principle, but nontradable for our investor. A typical example here is the valuation of (European) executive stock options. In both cases we obtain closed-form expressions for the exponential utility indifference value of the claim $B$ at all times $t \in[0, T]$. The key feature of our formulae is that the explicit form of the indifference value is preserved at any time $t$, except that the distortion power, which is shown to exist but is not explicitly determined, may now be random and depend on the contingent claim $B$ to be valued. To the best of our knowledge, this is the first explicit result on exponential utility indifference valuation in a setting with nonconstant and nondeterministic correlation. As another novelty, our general framework allows us to distinguish (via measurability conditions) between the settings of case (I) and case (II); this is impossible when $\rho$ and the instantaneous Sharpe ratio $\lambda$ of $S$ are constant, as in most of the existing literature. In Subsection 4.2 we discuss this and other issues in more detail.

In Section 5 we provide both intuitive and rigorous explanations for our results. We show that the value of the abstract optimization problem is monotonic in $|\rho|$. Because this value can be computed explicitly for constant $\rho$ and is continuous in the $\rho$-argument, interpolation implies that the basic structure is preserved for a random $\rho$. This explains why we can obtain our nice and explicit results. However, the precise interpretation of the above monotonicity is delicate, since it only holds when the ( $\rho$-dependent) probability measure $\hat{\mathbf{P}}(\rho)$ appearing in the abstract problem is kept fixed. A counterexample shows that the value of the original optimization problem under $\mathrm{P}$ may fail to be monotonic in $|\rho|$ if we allow $\hat{\mathrm{P}}(\rho)$ to vary with $\rho$, and we explain how keeping $\hat{\mathrm{P}}(\rho)$ fixed is linked to standard financial reasoning. 
For concreteness and ease of exposition, all our results are given for two correlated Brownian motions $W$ and $Y$. Finally, in Section 6 we briefly show how everything can be generalized to a multidimensional Itô process setting.

\section{Preliminaries}

\subsection{Model setup}

We work on a finite time interval $[0, T]$ for a fixed $T>0$ and a complete filtered probability space $(\Omega, g, \mathbb{G}, \mathrm{P})$. The filtration $\mathbb{G}=\left(g_{s}\right)_{0 \leq s \leq T}$ satisfies the usual conditions, has $g_{0}$ trivial, and $Y=\left(Y_{S}\right)_{0 \leq s \leq T}$ and $Y^{\perp}$ are two independent $(\mathbb{G}, \mathrm{P})$-Brownian motions. Unless otherwise mentioned, all processes and filtrations are indexed by $s \in[0, T]$, and we fix $t \in[0, T]$. For any process $X, \mathbb{F}^{X}=\left(\mathcal{F}_{S}^{X}\right)$ denotes the P-augmented filtration generated by $X$. For any filtration $\mathbb{F} \subseteq \mathbb{G}$, a process $X$ is called $\mathbb{F}$-predictable if it is measurable with respect to the $\mathbb{F}$-predictable $\sigma$-field on $[0, T] \times \Omega$, completed by the nullsets of (Lebesgue measure) $\otimes \mathrm{P}$. To simplify computations, we use the notation $\mathcal{E}(N)_{s, y}:=\exp \left(N_{y}-N_{s}-\frac{1}{2}\left(\langle N\rangle_{y}-\langle N\rangle_{s}\right)\right)$, $0 \leq s \leq y \leq T$, for a continuous $\mathbb{G}$-semimartingale $N$. Notions such as $L^{\infty}$ or 'almost surely' (a.s.) always refer to $\mathrm{P}$ (or any probability measure equivalent to $\mathrm{P}$ ).

The stochastic framework of our model consists of two Brownian motions $W$ and $Y$ with random instantaneous correlation $\rho$. To construct this, let $\rho=\left(\rho_{s}\right)$ be a $\mathbb{G}$-predictable process valued in $[-1,1]$ such that $|\rho|$ is bounded away from 1 (uniformly in $s$ and $\omega$ ), and define

$$
W_{s}:=\int_{0}^{s} \rho_{y} \mathrm{~d} Y_{y}+\int_{0}^{s} \sqrt{1-\rho_{y}^{2}} \mathrm{~d} Y_{y}^{\perp}, \quad 0 \leq s \leq T .
$$

In our financial market two assets are available for investing and going short: a risk-free bank account and a stock $S$. The instantaneous yield of the bank account is described by a deterministic spot interest rate function $r:[0, T] \rightarrow[0, \infty)$, which is bounded and Borelmeasurable. For ease of notation, we directly pass to discounted quantities, which means that we take $r \equiv 0$. (See Section 4 for more comments on this.) The (discounted) dynamics of the stock is given by

$$
\mathrm{d} S_{s}=\mu_{s} S_{s} \mathrm{~d} s+\sigma_{s} S_{s} \mathrm{~d} W_{s}, \quad 0 \leq s \leq T, S_{0}>0,
$$

where the drift $\mu$ and the volatility $\sigma$ are $\mathbb{G}$-predictable processes. We assume, for simplicity, that $\mu$ is bounded and $\sigma$ is bounded away from 0 and $\infty$. Hence, the instantaneous Sharpe ratio $\lambda:=\mu / \sigma$ is also bounded. We write

$$
\frac{\mathrm{d} S_{s}}{S_{s}}=\mu_{s} \mathrm{~d} s+\sigma_{s} \mathrm{~d} W_{s}=\sigma_{s} \mathrm{~d} \hat{W}_{s}, \quad 0 \leq s \leq T,
$$

and note that, by Girsanov's theorem, the processes

$$
\hat{W}_{s}:=W_{s}+\int_{0}^{s} \lambda_{y} \mathrm{~d} y \quad \text { and } \quad \hat{Y}_{s}:=Y_{s}+\int_{0}^{s} \rho_{y} \lambda_{y} \mathrm{~d} y, \quad 0 \leq s \leq T,
$$

are Brownian motions under the probability $\hat{\mathrm{P}} \approx \mathrm{P}$ on $\left(\Omega, g_{T}\right)$ given by

$$
\frac{\mathrm{d} \hat{\mathrm{P}}}{\mathrm{dP}}:=\mathcal{E}\left(-\int \lambda \mathrm{d} W\right)_{0, T} .
$$

In the terminology of [5], $\hat{\mathrm{P}}$ is the minimal martingale measure for $S$. 
Let $B$ be a bounded $g_{T}$-measurable random variable, interpreted as a contingent claim or payoff due at time $T$. To value $B$, we assume that our investor has an exponential utility function $U(x)=-\mathrm{e}^{-\gamma x}, x \in \mathbb{R}$, for a fixed $\gamma>0$. He starts at time $t \in[0, T]$ with initial capital $x_{t}$ and runs a self-financing strategy $\pi=\left(\pi_{s}\right)_{t \leq s \leq T}$ so that his wealth at time $s \in[t, T]$ is

$$
X_{s}^{x_{t}, \pi}=x_{t}+\int_{t}^{s} \frac{\pi_{y}}{S_{y}} \mathrm{~d} S_{y}=x_{t}+\int_{t}^{s} \pi_{y} \sigma_{y} \mathrm{~d} \hat{W}_{y},
$$

where $\pi$ represents the amount invested in $S$. The set $\mathcal{A}_{t}$ of admissible strategies on $[t, T]$ consists of all $\mathbb{G}$-predictable processes $\pi=\left(\pi_{s}\right)_{t \leq s \leq T}$ which satisfy $\int_{t}^{T} \pi_{s}^{2} \mathrm{~d} s<\infty$ a.s. and are such that

$$
\left(\exp \left(-\gamma \int_{t}^{s} \pi_{y} \sigma_{y} \mathrm{~d} \hat{W}_{y}\right)\right)_{t \leq s \leq T} \text { is of class }(D) \text { on }\left(\Omega, g_{T}, \mathbb{G}, \mathrm{P}\right),
$$

abbreviated by 'of P-class $(D)$ '. We define $V^{B}$ (and analogously $V^{0}$ ) for $t \in[0, T]$ and $x_{t}$ bounded $g_{t}$-measurable by

$$
\begin{aligned}
V_{t}^{B}\left(x_{t}\right) & :=\underset{\pi \in \mathcal{A}_{t}}{\operatorname{ess} \sup _{\mathrm{P}}\left[U\left(X_{T}^{x_{t}, \pi}-B\right) \mid g_{t}\right]} \\
& =-\exp \left(-\gamma x_{t}\right) \underset{\pi \in \mathcal{A}_{t}}{\operatorname{ess} \inf _{\mathrm{P}}} \mathrm{E}_{\mathrm{P}}\left[\exp \left(-\gamma \int_{t}^{T} \pi_{s} \sigma_{s} \mathrm{~d} \hat{W}_{s}+\gamma B\right) \mid g_{t}\right]
\end{aligned}
$$

using the fact that (bounded) $g_{t}$-measurable factors can be pulled out. Thus, $V_{t}^{B}\left(x_{t}\right)$ is the maximal expected utility the investor can achieve by starting at time $t$ with initial capital $x_{t}$, using some admissible strategy $\pi$, and paying out $B$ at time $T$.

Viewed over time $t, V^{B}(0)$ defined (up to a minus sign) by the essential infimum in (6) is the dynamic value process for the stochastic control problem associated to exponential utility maximization. One can show, by standard arguments, that $V^{B}(0)$ has a càdlàg version (which is right continuous with left limits) and then study its dynamic properties as a process; see, for instance, [1], [15], or [17]. However, our goal in this paper is rather to provide explicit or structural formulae for $V_{t}^{B}(0)$ with a fixed $t$.

Remark. Condition (5) is technically useful, but also has the following desirable implication. From an economic point of view, we should only allow strategies which are close in some sense to investments with finite credit lines, as Schachermayer [19] emphasized after his Definition 1.3. In our model any $\pi \in \mathcal{A}_{t}$ can be approximated in the following way. Consider a sequence $\left(\tau_{n}\right)_{n \in \mathbb{N}}$ of $\mathbb{G}$-stopping times increasing to $T$ stationarily and define a self-financing strategy $\pi^{(n)}=\pi \mathbf{1}_{\rrbracket t, \tau_{n} \rrbracket}$ by trading according to $\pi$ until $\tau_{n}$ and then putting all the capital into the bank account. This gives a terminal portfolio value of $x_{t}+\int_{t}^{\tau_{n}} \pi_{s} \sigma_{s} \mathrm{~d} \hat{W}_{s}$, leading to the individual utility $-\exp \left(-\gamma x_{t}-\gamma \int_{t}^{\tau_{n}} \pi_{s} \sigma_{s} \mathrm{~d} \hat{W}_{s}\right)$, which converges in $L^{1}(\mathrm{P})$ to the utility of the final value $X_{T}^{x_{t}, \pi}$ of the strategy $\pi$ due to (5). If we specifically choose

$$
\tau_{n}:=\inf \left\{s \in[t, T] \mid X_{s}^{x_{t}, \pi}-x_{t} \leq-n\right\} \wedge T, \quad n \in \mathbb{N},
$$

each of the approximating $\pi^{(n)}$ represents an investment with finite credit line. A similar approximation is used in Proposition 1 to find an upper bound for $V^{B}$, and the same class of strategies has been used in [12]. 
The indifference (seller) value $b_{t}\left(x_{t}\right)$ at time $t$ for $B$ is implicitly defined by

$$
V_{t}^{0}\left(x_{t}\right)=V_{t}^{B}\left(x_{t}+b_{t}\left(x_{t}\right)\right) .
$$

This says that the investor is indifferent between solely trading with initial capital $x_{t}$, versus trading with initial capital $x_{t}+b_{t}\left(x_{t}\right)$ but paying out $B$ at $T$. Our final goal is to find an explicit formula for $b_{t}\left(x_{t}\right)$. By (6),

$$
b_{t}=b_{t}\left(x_{t}\right)=\frac{1}{\gamma} \log \frac{V_{t}^{B}(0)}{V_{t}^{0}(0)}
$$

does not depend on $x_{t}$. This also shows that we are done once we have $V_{t}^{B}(0)$ explicitly, and so our focus henceforth lies on the optimization problem (6).

\subsection{Motivation}

Our goal is to find an explicit expression for

$$
-V_{t}^{B}(0)=\underset{\pi \in \mathcal{A}_{t}}{\operatorname{ess} \inf _{\mathrm{P}}} \mathrm{E}_{\mathrm{P}}\left[\exp \left(-\gamma \int_{t}^{T} \pi_{s} \sigma_{s} \mathrm{~d} \hat{W}_{s}+\gamma B\right) \mid g_{t}\right] .
$$

In Section 3 we study and solve an abstract martingale version of this problem, and we first explain how that formulation naturally arises out of (8). Since we only want to provide motivation, we ignore here all technical issues such as integrability, etc.

First suppose that $B \equiv 0$ and that $S$ is a (local) P-martingale; equivalently, $\mu=\lambda=0$ and $\hat{W}$ is a P-Brownian motion. Then the stochastic integral in (8) is a P-martingale, we minimize the expectation of a convex function of this, and so Jensen's inequality immediately tells us that the optimizer is $\pi^{\star} \equiv 0$ and that $V_{t}^{B}(0)=-1$.

In the general case where $S$ is a P-semimartingale, the idea is now to reduce (8) to the martingale case by writing

$$
-V_{t}^{B}(0)=\underset{\pi \in \mathcal{A}_{t}}{\operatorname{essinf}} \mathrm{E}_{\mathrm{P}^{\prime}}\left[\frac{Z_{t}^{\prime}}{Z_{T}^{\prime}} \exp \left(-\gamma \int_{t}^{T} \pi_{s} \sigma_{s} \mathrm{~d} \hat{W}_{s}+\gamma B\right) \mid g_{t}\right],
$$

where $Z^{\prime}$ is the P-density process of some fixed measure $\mathrm{P}^{\prime}$ (not depending on $\pi$ ) under which $S$ or $\hat{W}$ is a local martingale. To choose a good $\mathrm{P}^{\prime}$, one might be tempted by the duality results of [3] to take the minimal entropy martingale measure $Q^{E}$, because its density $Z_{T}^{E}$ is up to a constant the exponential of a stochastic integral of $S$. However, this is not true for the density $Z_{t}^{E}$ on $g_{t}$, and it is in general also very difficult to find $Q^{E}$ explicitly in any given model. Because we want explicit formulae, we need $Z_{t}^{\prime} / Z_{T}^{\prime}$ as explicitly as possible. Now any equivalent local martingale measure $\mathrm{P}^{\prime}$ has a $\mathrm{P}$-density process of the form $Z^{\prime}=\mathcal{E}\left(-\int \lambda \mathrm{d} W\right) \mathcal{E}(N)$ for some local P-martingale $N$ orthogonal to $W$, and inserting this expression for $Z^{\prime}$ into (9) gives, after a straightforward calculation,

$$
\begin{aligned}
& \mathrm{E}_{\mathrm{P}}\left[\exp \left(-\gamma \int_{t}^{T} \pi_{s} \sigma_{s} \mathrm{~d} \hat{W}_{s}+\gamma B\right) \mid g_{t}\right] \\
& \quad=\mathrm{E}_{\hat{\mathrm{P}}}\left[\exp \left(-\int_{t}^{T}\left(\gamma \pi_{s} \sigma_{s}-\lambda_{s}\right) \mathrm{d} \hat{W}_{s}+\gamma B-\frac{1}{2} \int_{t}^{T} \lambda_{s}^{2} \mathrm{~d} s\right) \mid g_{t}\right] .
\end{aligned}
$$

The minimal martingale measure $\hat{P}$ from (4) appears naturally in this way, and it has the enormous benefit that its density $\hat{Z}=\mathcal{E}\left(-\int \lambda \mathrm{d} W\right)$ is completely explicit. Combining (10) 
with (8) gives

$$
-V_{t}^{B}(0)=\underset{\pi \in \mathcal{A}_{t}}{\operatorname{ess} \inf _{\hat{\mathrm{P}}}} \mathrm{E}_{\hat{\mathrm{C}}}\left[\exp \left(-\int_{t}^{T}\left(\gamma \pi_{s} \sigma_{s}-\lambda_{s}\right) \mathrm{d} \hat{W}_{s}+\bar{B}\right) \mid g_{t}\right],
$$

and we can recognize this as a 'martingale version' of (8) with an artificial random endowment

$$
\bar{B}:=\gamma B-\frac{1}{2} \int_{t}^{T} \lambda_{s}^{2} \mathrm{~d} s
$$

Note that in the genuine semimartingale case, $\lambda \not \equiv 0$, the quantity $\bar{B}$ appears even if the claim $B$ is 0 . Hence, there is no simplification from assuming that $B \equiv 0$, and so we do not discuss this case separately.

\section{The main (but abstract) result}

\subsection{An explicit formula for an optimization problem}

This section contains the main mathematical contribution of this paper. We derive an explicit formula for the value of the following optimization problem:

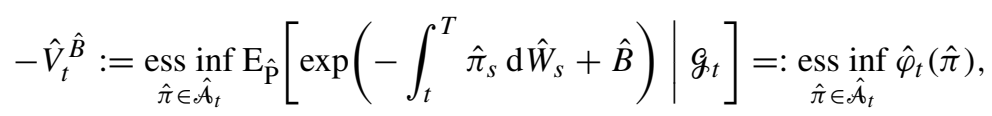

where $\hat{\mathcal{A}}_{t}$ consists of all $\mathbb{G}$-predictable $\hat{\pi}=\left(\hat{\pi}_{s}\right)_{t \leq s \leq T}$ satisfying $\int_{t}^{T} \hat{\pi}_{s_{\hat{\lambda}}}^{2} \mathrm{~d} s<\infty$ a.s. and such that $\left(\exp \left(-\int_{t}^{s} \hat{\pi}_{y} \mathrm{~d} \hat{W}_{y}\right)\right)_{t \leq s \leq T}$ is of $\hat{\mathrm{P}}$-class $(D)$. Here $\hat{B}$ is a bounded $\hat{\mathscr{H}}_{T}$-measurable random variable, where $\hat{\mathbb{H}}=\left(\hat{\mathscr{H}}_{S}\right) \subseteq \mathbb{G}$ is a filtration such that the $\hat{\mathrm{P}}$-Brownian motion $\hat{Y}$ from (3) has the representation property in $\hat{\mathbb{H}}$. This means that any $(\hat{\mathbb{H}}, \hat{\mathrm{P}})$-martingale $L$ is of the form $L=L_{0}+\int \zeta \mathrm{d} \hat{Y}$ for an $\hat{\mathbb{H}}$-predictable $\zeta$ with $\int_{0}^{T} \zeta_{s}^{2} \mathrm{~d} s<\infty$ a.s. The assumption that $\hat{B} \in L^{\infty}\left(\hat{\mathscr{H}}_{T}\right)$ is slightly weaker than $\hat{B} \in L^{\infty}\left(\mathcal{F}_{T}^{\hat{Y}}\right)$, and the two different applications in Section 4 will make it clear why this is useful. It is worth pointing out that all the subsequent arguments only involve the filtration $\hat{H}$; this is the reason why we can formulate our model with a general filtration $\mathbb{G} \supseteq \mathbb{F}^{Y, Y^{\perp}}$ such that $Y$ and $Y^{\perp}$ are $(\mathbb{G}, \mathrm{P})$-Brownian motions.

While the idea of considering a problem like (13) has been motivated in Subsection 2.2 from (8), it is not clear at this stage how $\hat{B}$ and especially $\hat{\mathbb{H}}$ arise. This will become clearer in Section 4 from the applications. However, we already point out that $\hat{B}$ and the artificial claim $\bar{B}=\gamma B-\frac{1}{2} \int_{t}^{T} \lambda_{s}^{2} \mathrm{~d} s$ from (12) can be different.

Theorem 1. Under the above assumptions, set

$$
\underline{\delta}_{t}:=\inf _{s \in[t, T]} \frac{1}{\left\|1-\rho_{s}^{2}\right\|_{L^{\infty}}} \text { and } \quad \bar{\delta}_{t}:=\sup _{s \in[t, T]}\left\|\frac{1}{1-\rho_{s}^{2}}\right\|_{L^{\infty}} .
$$

Then there exists a $\mathcal{g}_{t}$-measurable random variable $\delta_{t}^{\hat{B}}$ with values in $\left[\underline{\delta}_{t}, \bar{\delta}_{t}\right]$ such that

$$
-\hat{V}_{t}^{\hat{B}}(\omega)=\left.\left(\mathrm{E}_{\hat{\mathrm{P}}}\left[\exp (\hat{B})^{1 / \delta} \mid \hat{\mathcal{H}}_{t}\right](\omega)\right)^{\delta}\right|_{\delta=\delta_{t}^{\hat{B}}(\omega)}
$$

for almost all $\omega \in \Omega$.

The right-hand side of (15) is understood as follows. We compute, for fixed $\delta$, (a version of) the $\left(\hat{\mathscr{H}}_{t}, \hat{\mathrm{P}}\right)$-conditional expectation of $\exp (\hat{B})^{1 / \delta}$, evaluate that (version) in the given $\omega$, and then insert for $\delta$ the value $\delta_{t}^{\hat{B}}(\omega)$. 
Before we actually prove Theorem 1 , we provide an outline of the proof here. The key idea is to find a family of processes $Z^{(\hat{\pi})}$ with

$$
Z_{T}^{(\hat{\pi})}=\exp \left(-\int_{t}^{T} \hat{\pi}_{s} \mathrm{~d} \hat{W}_{s}+\hat{B}\right)
$$

and such that $Z^{(\hat{\pi})}$ is a $(\mathbb{G}, \hat{\mathrm{P}})$-submartingale for every $\hat{\pi} \in \hat{\mathcal{A}}_{t}$ and a $(\mathbb{G}, \hat{\mathrm{P}})$-martingale for some $\hat{\pi}=\hat{\pi}^{\star} \in \hat{\mathcal{A}}_{t}$. If we can do this, the same argument as in Subsection 2.2 easily shows that the essential infimum in (13) is attained for $\hat{\pi}^{\star}$.

To find such a family $Z^{(\hat{\pi})}$, we need a good representation for $\mathrm{e}^{\hat{B}}$, and the multiplicative form of (16) might suggest that we write $\exp (\hat{B})$ as the final value of some stochastic exponential martingale. But unless we believe that $\hat{\pi}^{\star} \equiv 0$ happens to be optimal, $\mathrm{e}^{\hat{B}}=Z_{T}^{(0)}$ should be the final value of a $(\mathbb{G}, \hat{\mathrm{P}})$-submartingale rather than a $(\mathbb{G}, \hat{\mathrm{P}})$-martingale. Again in view of the multiplicative structure, the simplest way to transform a positive martingale into a submartingale is to raise it to a power bigger than 1. Fixing a constant $\delta \geq 1$ to be specified later and using $\hat{B} \in L^{\infty}\left(\hat{\mathscr{H}}_{T}\right)$, we thus write

$$
\mathrm{e}^{\hat{B}}=\exp \left(\frac{\hat{B}}{\delta}\right)^{\delta}=\left(c_{t} \mathcal{E}(L)_{t, T}\right)^{\delta}, \quad c_{t}:=\mathrm{E}_{\hat{\mathrm{P}}}\left[\exp \left(\frac{\hat{B}}{\delta}\right) \mid \hat{\mathscr{H}}_{t}\right]
$$

for a $B M O(\hat{\mathbb{H}}, \hat{\mathrm{P}})$-martingale $L$. (More precisely, the positive $(\hat{\mathbb{H}}, \hat{\mathrm{P}})$-martingale with final value $\exp (\hat{B} / \delta)$ is uniformly bounded away from 0 and $\infty$, and, thus, its stochastic logarithm $L$ is in $B M O$.) By the representation property of $\hat{Y}$ in $\hat{\mathbb{H}}, L$ is of the form

$$
L=\int \zeta \mathrm{d} \hat{Y} \text { for an } \hat{\mathbb{H}} \text {-predictable } \zeta \text { with } \mathrm{E}_{\hat{\mathrm{P}}}\left[\int_{0}^{T} \zeta_{s}^{2} \mathrm{~d} s\right]<\infty .
$$

So $L$ is a $B M O(\mathbb{G}, \hat{\mathrm{P}})$-martingale, too, and combining (16) and (17) gives

$$
\begin{aligned}
Z_{T}^{(\hat{\pi})} & =c_{t}^{\delta}\left(\mathcal{E}(L)_{t, T}\right)^{\delta} \exp \left(-\int_{t}^{T} \hat{\pi}_{s} \mathrm{~d} \hat{W}_{s}\right) \\
& =c_{t}^{\delta} \mathcal{E}(\delta L)_{t, T} \mathcal{E}\left(-\int \hat{\pi} \mathrm{d} \hat{W}\right)_{t, T} \exp \left(\frac{1}{2} \int_{t}^{T}\left(\left(\delta^{2}-\delta\right) \zeta_{s}^{2}+\hat{\pi}_{s}^{2}\right) \mathrm{d} s\right) .
\end{aligned}
$$

Using Yor's formula, (17), and $\mathrm{d}\langle\hat{Y}, \hat{W}\rangle_{s}=\rho_{s} \mathrm{~d} s$ yields

$$
\begin{aligned}
\mathscr{E}(\delta L) \mathcal{E}\left(-\int \hat{\pi} \mathrm{d} \hat{W}\right) & =\mathcal{E}\left(\delta L-\int \hat{\pi} \mathrm{d} \hat{W}-\left\langle\delta L, \int \hat{\pi} \mathrm{d} \hat{W}\right\rangle\right) \\
& =M^{(\hat{\pi})} \exp \left(-\int \delta \zeta_{s} \hat{\pi}_{s} \rho_{s} \mathrm{~d} s\right)
\end{aligned}
$$

with the local $(\mathbb{G}, \hat{\mathrm{P}})$-martingale

$$
M^{(\hat{\pi})}:=\mathcal{E}\left(\delta L-\int \hat{\pi} \mathrm{d} \hat{W}\right)=\mathcal{E}\left(\int \delta \zeta \mathrm{d} \hat{Y}-\int \hat{\pi} \mathrm{d} \hat{W}\right),
$$

and putting everything together and completing squares leads us to define

$$
Z_{s}^{(\hat{\pi})}:=c_{t}^{\delta} M_{t, s}^{(\hat{\pi})} \exp \left(\frac{1}{2} \int_{t}^{s}\left(\left(\hat{\pi}_{y}-\delta \zeta_{y} \rho_{y}\right)^{2}+\zeta_{y}^{2} \delta\left(\delta\left(1-\rho_{y}^{2}\right)-1\right)\right) \mathrm{d} y\right) \quad \text { for } t \leq s \leq T .
$$


This gives (16) by construction, and if $\rho$ is constant, choosing $\delta:=1 /\left(1-\rho^{2}\right)$ ensures that the integrand in (20) is always nonnegative and vanishes for $\hat{\pi}^{\star}=\delta \zeta \rho$. Hence, $Z^{(\hat{\pi})}$ is then, on $[t, T]$, a local $(\mathbb{G}, \hat{\mathrm{P}})$-submartingale for every $\hat{\pi}$ and a local $(\mathbb{G}, \hat{\mathrm{P}})$-martingale for $\hat{\pi}^{\star}$. Apart from integrability issues, we have thus achieved our goal in that case.

In general, $\rho$ is not constant. Then we choose one $\delta$ for the submartingale property of $Z^{(\hat{\pi})}$ for all $\hat{\pi}$ and another $\delta$ for the martingale property of $Z^{\left(\hat{\pi}^{\star}\right)}$. This gives an upper bound and a lower bound for $\hat{V}_{t}^{\hat{B}}$, and Theorem 1 is obtained by interpolation. The detailed proof is given in the next subsection.

Remark. The attentive reader may have noticed that we only give results on the value of the optimization problem, and may argue that, for hedging or investing purposes, one would also like to know the optimal strategy explicitly. While this is a valid point, it is a well-known unfortunate fact that this problem is notoriously difficult even in quite specific (e.g. Markovian) settings. We hope to address this question in subsequent work, as it goes beyond the scope of the present paper.

\subsection{Proof of Theorem 1}

The argument for Theorem 1 follows the outline given in Subsection 3.1. We suppose throughout that the assumptions of Theorem 1 hold and first derive an upper bound for $\hat{V}_{t}^{\hat{B}}$. Recall $\hat{\varphi}_{t}(\hat{\pi})$ from (13) and $\bar{\delta}_{t}$ from (14).

Proposition 1. For all $\hat{\pi} \in \hat{\mathcal{A}}_{t}$, we have

$$
\hat{\varphi}_{t}(\hat{\pi}) \geq \mathrm{E}_{\hat{\mathrm{P}}}\left[\exp \left(\frac{\hat{B}}{\bar{\delta}_{t}}\right) \mid \hat{\mathscr{H}}_{t}\right]^{\bar{\delta}_{t}} \text { a.s. }
$$

Proof. We use the reasonings and notation from Subsection 3.1 with $\delta:=\bar{\delta}_{t}$.

1. First suppose that $\int_{t}^{T} \hat{\pi}_{s}^{2} \mathrm{~d} s$ is uniformly bounded. Then $\int \hat{\pi} \mathrm{d} \hat{W}$ is a $B M O(\mathbb{G}, \hat{\mathrm{P}})$-martingale like $L=\int \zeta \mathrm{d} \hat{Y}$ from (18) and, hence, by Theorem 2.3 of [14], $M^{(\hat{\pi})}=\mathcal{E}\left(\delta L-\int \hat{\pi} \mathrm{d} \hat{W}\right)$ from $(19)$ is a $(\mathbb{G}, \hat{\mathrm{P}})$-martingale. The choice $\delta=\bar{\delta}_{t}$ implies that the integrand in (20) is nonnegative and, thus, $Z^{(\hat{\pi})}$ is a $(\mathbb{G}, \hat{\mathrm{P}})$-submartingale; in fact, integrability follows via (16) because $Z_{T}^{(\hat{\pi})}=\exp \left(-\int_{t}^{T} \hat{\pi}_{s} \mathrm{~d} \hat{W}_{s}+\hat{B}\right)$ is in $L^{1}(\hat{\mathrm{P}})$ since $\int \hat{\pi} \mathrm{d} \hat{W}$ is in $B M O$. Thus, (13), (16), (17), and (20) yield

$$
\hat{\varphi}_{t}(\hat{\pi})=\mathrm{E}_{\hat{\mathrm{P}}}\left[Z_{T}^{(\hat{\pi})} \mid g_{t}\right] \geq Z_{t}^{(\hat{\pi})}=\mathrm{E}_{\hat{\mathrm{P}}}\left[\exp \left(\frac{\hat{B}}{\bar{\delta}_{t}}\right) \mid \hat{\mathscr{H}}_{t}\right]^{\bar{\delta}_{t}} \quad \text { a.s. }
$$

2. In general, we define a localizing sequence by

$$
\tau_{n}:=\inf \left\{s \in[t, T] \text { such that } \int_{t}^{s} \hat{\pi}_{y}^{2} \mathrm{~d} y \geq n\right\} \wedge T, \quad n \in \mathbb{N},
$$

and set $\hat{\pi}^{(n)}:=\hat{\pi} \mathbf{1}_{\rrbracket t, \tau_{n} \rrbracket} \in \hat{\mathcal{A}}_{t}$. Applying step 1 to $\hat{\pi}^{(n)}$ then gives

$$
\hat{\varphi}_{t}\left(\hat{\pi}^{(n)}\right) \geq \mathrm{E}_{\hat{\mathrm{P}}}\left[\exp \left(\frac{\hat{B}}{\bar{\delta}_{t}}\right) \mid \hat{\mathscr{H}}_{t}\right]^{\bar{\delta}_{t}} \text { a.s. }
$$

Because $\left(\exp \left(-\int_{t}^{s} \hat{\pi}_{y} \mathrm{~d} \hat{W}_{y}\right)\right)_{t \leq s \leq T}$ is of $\hat{\mathrm{P}}$-class $(D)$ and $\hat{B}$ is bounded, the sequence

$$
\left(Z_{T}^{\left(\hat{\pi}^{(n)}\right)}\right)_{n \in \mathbb{N}}=\left(\exp \left(-\int_{t}^{T} \hat{\pi}_{s}^{(n)} \mathrm{d} \hat{W}_{s}+\hat{B}\right)\right)_{n \in \mathbb{N}}
$$


is $\hat{\mathrm{P}}$-uniformly integrable and converges a.s. to $Z_{T}^{(\hat{\pi})}$. Hence, the conditioned random variables $\hat{\varphi}_{t}\left(\hat{\pi}^{(n)}\right)=\mathrm{E}_{\hat{\mathrm{P}}}\left[Z_{T}^{\left(\hat{\pi}^{(n)}\right)} \mid g_{t}\right], n \in \mathbb{N}$, converge to $\hat{\varphi}_{t}(\hat{\pi})$ in $L^{1}(\hat{\mathrm{P}})$ and, therefore, also a.s. along a subsequence. This concludes the proof in view of (21).

The next result entails a lower bound for $\hat{V}_{t}^{\hat{B}}$. Recall $\underline{\delta}_{t}$ from (14).

Proposition 2. Define $\hat{\pi}^{\star}=\left(\hat{\pi}_{s}^{\star}\right)_{t \leq s \leq T}$ by

$$
\hat{\pi}_{s}^{\star}:=\left(\rho_{s} \underline{\delta}_{t}+\sqrt{\rho_{s}^{2} \underline{\delta}_{t}^{2}+\underline{\delta}_{t}-\underline{\delta}_{t}^{2}}\right) \zeta_{s}, \quad t \leq s \leq T,
$$

where $\zeta$ is now determined as in (17) and (18) with $\delta:=\underline{\delta}_{t}$. Then we have

$$
\hat{\varphi}_{t}\left(\hat{\pi}^{\star}\right)=\mathrm{E}_{\hat{\mathrm{P}}}\left[\exp \left(\frac{\hat{B}}{\underline{\delta}_{t}}\right) \mid \hat{\mathscr{H}}_{t}\right]^{\underline{\delta}_{t}} \text { a.s. and } \hat{\pi}^{\star} \in \hat{\mathcal{A}}_{t} .
$$

To be more precise, $\hat{\pi}_{s}^{\star}$ is, for any $s \in[t, T]$, defined by (22) on the set

$$
\tilde{\Omega}_{s}=\left\{\omega \in \Omega \mid \frac{1}{1-\rho_{s}^{2}(\omega)} \geq \underline{\delta}_{t}\right\},
$$

which has (P-and $\hat{\mathrm{P}}_{-}$) probability 1 . For $\omega \notin \tilde{\Omega}_{s}$, we set $\hat{\pi}_{s}^{\star}(\omega):=0$. By the definition of $\underline{\delta}_{t}$, the expression under the square root in (22) is nonnegative, and $\hat{\pi}^{\star}$ is $\mathbb{G}$-predictable.

Proof of Proposition 2. We use the notation of Subsection 3.1 with $\delta:=\underline{\delta}_{t}$, and first show the equality in (23). Because $\hat{\pi}^{\star}$ in (22) is chosen to make the integrand in (20) vanish, from (20) we obtain

$$
Z_{T}^{\left(\hat{\pi}^{\star}\right)}=c_{t}^{\underline{\delta}_{t}} M_{t, T}^{\left(\hat{\pi}^{\star}\right)}=\mathrm{E}_{\hat{\mathrm{P}}}\left[\exp \left(\frac{\hat{B}}{\underline{\delta}_{t}}\right) \mid \hat{\mathscr{H}}_{t}\right]^{\underline{\delta}_{t}} \mathcal{E}\left(N^{\left(\hat{\pi}^{\star}\right)}\right)_{t, T}
$$

with $N^{\left(\hat{\pi}^{\star}\right)}:=\int \underline{\delta}_{t} \zeta \mathrm{d} \hat{Y}-\int \hat{\pi}^{\star} \mathrm{d} \hat{W}$. An easy computation using (22) yields

$$
\left\langle N^{\left(\hat{\pi}^{\star}\right)}\right\rangle_{s}-\left\langle N^{\left(\hat{\pi}^{\star}\right)}\right\rangle_{t}=\underline{\delta}_{t} \int_{t}^{s} \zeta_{y}^{2} \mathrm{~d} y=\underline{\delta}_{t}\left(\langle L\rangle_{s}-\langle L\rangle_{t}\right), \quad t \leq s \leq T,
$$

and so $N^{\left(\hat{\pi}^{\star}\right)}$ is, like $\int \zeta \mathrm{d} \hat{Y}=L$, a $B M O(\mathbb{G}, \hat{\mathrm{P}})$-martingale; see below (18). We conclude, by Theorem 2.3 of [14], that $M^{\left(\hat{\pi}^{\star}\right)}=\mathcal{E}\left(N^{\left(\hat{\pi}^{\star}\right)}\right)$ is a $(\mathbb{G}, \hat{\mathrm{P}})$-martingale, and so (13), (16), and (24) yield the equality in (23).

To prove $\hat{\pi}^{\star} \in \hat{\mathcal{A}}_{t}$, we first note that $\mathrm{E}_{\hat{\mathrm{P}}}\left[\int_{t}^{T} \zeta_{s}^{2} \mathrm{~d} s\right]<\infty$ from (18) implies that

$$
\mathrm{E}_{\hat{\mathrm{P}}}\left[\int_{t}^{T}\left|\hat{\pi}_{s}^{\star}\right|^{2} \mathrm{~d} s\right]<\infty,
$$

by (22). To show that $\left(\exp \left(-\int_{t}^{s} \hat{\pi}_{y}^{\star} \mathrm{d} \hat{W}_{y}\right)\right)_{t \leq s \leq T}$ is of $\hat{\mathrm{P}}$-class $(D)$, we observe that (18) and (25) yield

$$
\exp \left(-\int_{t}^{s} \hat{\pi}_{y}^{\star} \mathrm{d} \hat{W}_{y}\right)=\exp \left(N_{s}^{\left(\hat{\pi}^{\star}\right)}-N_{t}^{\left(\hat{\pi}^{\star}\right)}-\underline{\delta}_{t} \int_{t}^{s} \zeta_{y} \mathrm{~d} \hat{Y}_{y}\right)=M_{t, s}^{\left(\hat{\pi}^{\star}\right)} \mathcal{E}(L)_{t, s}^{-\underline{\delta}_{t}}
$$

for $s \in[t, T]$, and the process $\mathcal{E}(L)^{-\delta_{t}}$ is bounded because (17) gives

$$
\mathscr{E}(L)_{t, s}=\frac{\mathrm{E}_{\hat{\mathrm{P}}}\left[\exp \left(\hat{B} / \underline{\delta}_{t}\right) \mid \hat{\mathscr{H}}_{s}\right]}{\mathrm{E}_{\hat{\mathrm{P}}}\left[\exp \left(\hat{B} / \underline{\delta}_{t}\right) \mid \hat{\mathscr{H}}_{t}\right]}, \quad t \leq s \leq T,
$$

and $\hat{B}$ is bounded. Moreover, $M^{\left(\hat{\pi}^{\star}\right)}$ as a $(\mathbb{G}, \hat{\mathrm{P}})$-martingale is of $\hat{\mathrm{P}}$-class $(D)$ and, hence, so is $\left(\exp \left(-\int_{t}^{s} \hat{\pi}_{y}^{\star} \mathrm{d} \hat{W}_{y}\right)\right)_{t \leq s \leq T}$. Thus, $\hat{\pi}^{\star}$ is in $\hat{\mathcal{A}}_{t}$, which concludes the proof. 
Remark. The choice of $\hat{\pi}^{\star}$ in (22) deserves a comment. As we have seen in the proof of Proposition 2, it ensures that the integrand

$$
\left(\underline{\delta}_{t} \rho_{s} \zeta_{s}-\hat{\pi}_{s}^{\star}\right)^{2}+\zeta_{s}^{2} \underline{\delta}_{t}\left(\underline{\delta}_{t}\left(1-\rho_{s}^{2}\right)-1\right)
$$

in (20) (with $\delta=\underline{\delta}_{t}$ and $\hat{\pi}=\hat{\pi}^{\star}$ ) vanishes identically. But, for fixed $s \in[t, T]$ and $\omega \in \Omega$, (26) is a quadratic function in $\hat{\pi}_{s}^{\star}(\omega)$, and requiring it to be 0 for each $s$ does not determine the process $\hat{\pi}^{\star}$ uniquely. In fact, Proposition 2 remains true if $\hat{\pi}^{\star}$ is replaced by $\hat{\pi}^{\eta}$ with

$$
\hat{\pi}_{s}^{\eta}:=\left(\rho_{s} \underline{\delta}_{t}+\eta_{s} \sqrt{\rho_{s}^{2} \underline{\delta}_{t}^{2}+\underline{\delta}_{t}-\underline{\delta}_{t}^{2}}\right) \zeta_{s}, \quad t \leq s \leq T,
$$

for any $\mathbb{G}$-predictable process $\eta$ on $[t, T]$ with values in $\{-1,1\}$.

Now suppose that we replace $\hat{\pi}^{\star}$ by $\hat{\pi}^{\star \star}$ with $\hat{\pi}_{s}^{\star \star}:=\underline{\delta}_{t} \rho_{s} \zeta_{s}, s \in[t, T]$, which minimizes (26) pointwise and makes it nonpositive. Then we obtain

$$
\hat{\varphi}_{t}\left(\hat{\pi} \hat{\pi}^{\star \star}\right)=\mathrm{E}_{\hat{\mathrm{P}}}\left[Z_{T}^{\left(\hat{\pi}^{\star \star}\right)} \mid g_{t}\right] \leq \mathrm{E}_{\hat{\mathrm{P}}}\left[\mathrm{E}_{\hat{\mathrm{P}}}\left[\exp \left(\frac{\hat{B}}{\underline{\delta}_{t}}\right) \mid \hat{\mathscr{H}}_{t}\right]^{\delta_{t}} M_{t, T}^{\left(\hat{\pi}^{\star \star}\right)} \mid g_{t}\right]=\hat{\varphi}_{t}\left(\hat{\pi}^{\star}\right),
$$

using the fact that $M^{\left(\hat{\pi}^{\star \star}\right)}$ is, like $M^{\left(\hat{\pi}^{\star}\right)}$, a $(\mathbb{G}, \hat{\mathrm{P}})$-martingale. Similar arguments as for $\hat{\pi}^{\star}$ also yield $\hat{\pi}^{\star \star} \in \hat{\mathcal{A}}_{t}$, and we even obtain $\hat{\varphi}_{t}\left(\hat{\pi}^{\star \star}\right)<\hat{\varphi}_{t}\left(\hat{\pi}^{\star}\right)$ on a set $A \in g_{t}$ with $\hat{\mathrm{P}}[A]>0$ if $\int_{t}^{T} \zeta_{s}^{2}\left(\underline{\delta}_{t}\left(1-\rho_{s}^{2}\right)-1\right) \mathrm{d} s$ is nonzero with positive probability. This shows that the lower bound

$$
-\hat{\varphi}_{t}\left(\hat{\pi}^{\star}\right) \leq-\underset{\hat{\pi} \in \hat{\mathcal{A}}_{t}}{\operatorname{essinf}} \hat{\varphi}_{t}(\hat{\pi})=\hat{V}_{t}^{\hat{B}} \quad \text { a.s. }
$$

entailed by Proposition 2 need not be sharp. Nevertheless, we work with $\hat{\pi}^{\star}$ and not with $\hat{\pi}^{\star \star}$, because $\hat{\varphi}_{t}\left(\hat{\pi}^{\star}\right)$ has the nice representation (23) which allows us to obtain an explicit expression for $\hat{V}_{t}^{\hat{B}}$; see the interpolation argument below. The sharper bound given via $\hat{\pi}^{\star \star}$ is not explicit enough to give this result. We remark that if $\rho$ is constant, $\hat{\pi}^{\star \star}$ and $\hat{\pi}^{\star}$ coincide and (27) holds with equality; compare Propositions 1 and 2.

As announced, we now prove Theorem 1 by an interpolation argument.

Proof of Theorem 1. Define $f(\cdot, \cdot):\left[\underline{\delta}_{t}, \bar{\delta}_{t}\right] \times \Omega \rightarrow \mathbb{R}$ by

$$
f(\delta, \omega):=\left(\mathrm{E}_{\hat{\mathrm{P}}}\left[\exp (\hat{B})^{1 / \delta} \mid \hat{\mathscr{H}}_{t}\right](\omega)\right)^{\delta}, \quad(\delta, \omega) \in\left[\underline{\delta}_{t}, \bar{\delta}_{t}\right] \times \Omega .
$$

Because $\hat{B}$ is bounded, dominated convergence and Jensen's inequality imply that $f$ admits a version which is continuous and nonincreasing in $\delta$ for each fixed $\omega \in \Omega$. We use this version in the sequel. From Propositions 1 and 2, we already know that

$$
f\left(\bar{\delta}_{t}, \omega\right) \leq-\hat{V}_{t}^{\hat{B}}(\omega) \leq f\left(\underline{\delta}_{t}, \omega\right) \text { for almost all } \omega \in \Omega .
$$

By the intermediate value theorem, the set

$$
\Delta(\omega):=\left\{\delta \in\left[\underline{\delta}_{t}, \bar{\delta}_{t}\right] \mid f(\delta, \omega)=-\hat{V}_{t}^{\hat{B}}(\omega)\right\}
$$

is thus nonempty for almost all $\omega \in \Omega$. Define $\delta_{t}^{\hat{B}}: \Omega \rightarrow\left[\underline{\delta}_{t}, \bar{\delta}_{t}\right]$ by

$$
\delta_{t}^{\hat{B}}(\omega):=\sup \Delta(\omega), \quad \omega \in \Omega,
$$


setting $\delta_{t}^{\hat{B}}(\cdot):=\left(\underline{\delta}_{t}+\bar{\delta}_{t}\right) / 2$ on the nullset $\{\omega \in \Omega \mid \Delta(\omega)=\varnothing\}$. By the continuity of $f$ in its first argument, $\Delta(\omega)$ is closed in $\mathbb{R}$ for all $\omega \in \Omega$, and we obtain, for almost all $\omega \in \Omega$,

$$
f\left(\delta_{t}^{\hat{B}}(\omega), \omega\right)=-\hat{V}_{t}^{\hat{B}}(\omega)
$$

It remains to prove that the mapping $\omega \mapsto \delta_{t}^{\hat{B}}(\omega)$ is $g_{t}$-measurable. Because $f$ is nonincreasing and owing to (28) and (29), we have, for any $a \in\left[\underline{\delta}_{t}, \bar{\delta}_{t}\right]$, up to a null set,

$$
\begin{aligned}
\left\{\omega \in \Omega \mid \delta_{t}^{\hat{B}}(\omega)<a\right\} & =\left\{\omega \in \Omega \mid f\left(\delta_{t}^{\hat{B}}(\omega), \omega\right)>f(a, \omega)\right\} \\
& =\left\{\omega \in \Omega \mid-\hat{V}_{t}^{\hat{B}}(\omega)>f(a, \omega)\right\} \\
& =\bigcup_{q \in \mathbb{Q}}\left\{\omega \mid-\hat{V}_{t}^{\hat{B}}(\omega)>q\right\} \cap\{\omega \mid q>f(a, \omega)\} .
\end{aligned}
$$

The last set is in $g_{t}$ since $\hat{V}_{t}^{\hat{B}}$ and $f(a, \cdot)$ for a fixed $a \in\left[\underline{\delta}_{t}, \bar{\delta}_{t}\right]$ are $g_{t}$-measurable. Since $g_{t}$ is complete, we have $\left\{\omega \in \Omega \mid \delta_{t}^{\hat{B}}(\omega)<a\right\} \in g_{t}$ for every $a \in \mathbb{R}$, and so $\delta_{t}^{\hat{B}}(\cdot)$ is $g_{t}$-measurable. This completes the proof.

\section{Applications in two settings}

\subsection{Explicit formulae for the indifference value}

Our goal in this section is to find explicit formulae for $V_{t}^{B}(0)$ in (6) or (11) in two different settings. This will be achieved by applying Theorem 1 and will also yield explicit results for the indifference value $b_{t}$ via (7). We recall $W$ and $\hat{Y}$ from (1) and (3) and write, for brevity,

$$
\mathbb{F}=\left(\mathcal{F}_{s}\right) \text { for } \mathbb{F}^{W}, \quad \mathbb{Y}=\left(\mathcal{y}_{s}\right) \text { for } \mathbb{F}^{Y}, \quad \hat{\mathbb{Y}}=\left(\hat{\mathcal{Y}}_{s}\right) \text { for } \mathbb{F}^{\hat{Y}} .
$$

If $\rho \lambda$ is $\mathbb{Y}$-predictable then $\hat{Y}$ from (3) is $\mathbb{Y}$-adapted and, hence, $\hat{\mathbb{Y}} \subseteq \mathbb{Y}$. In general, however, none of the above three filtrations contains any other.

Theorem 1 gives us the freedom to specify the artificial endowment $\hat{B}$, but also the task of finding a filtration $\hat{\mathbb{H}}$ such that $\hat{B}$ is $\hat{\mathscr{H}}_{T}$-measurable and $\hat{Y}$ has the representation property in $\hat{\mathbb{H}}$. Comparing (11) with (13) suggests choosing $\hat{B}=\bar{B}=\gamma B-\frac{1}{2} \int_{t}^{T} \lambda_{s}^{2} \mathrm{~d} s$. In a first application, we do this, and moreover we set $\hat{\mathbb{H}}=\mathbb{Y}$ and assume that $B$ is $\mathcal{y}_{T}$-measurable and $\lambda$ is $\mathbb{Y}$-predictable to ensure that $\hat{B}$ is $\hat{\mathscr{H}}_{T}$-measurable. We will later see in the proof of Theorem 2 that we also need to assume that $\rho$ is $\mathbb{Y}$-predictable to guarantee that $\hat{Y}$ has the representation property in $\mathbb{Y}$.

For our second application, we choose $\hat{B}=\gamma B$ and assume that $\frac{1}{2} \int_{t}^{T} \lambda_{s}^{2} \mathrm{~d} s$ is replicable by trading in $S$. This is satisfied if $\lambda$ is $\mathbb{F}$-predictable, as we will see in the proof of Theorem 3 . In this case we, moreover, set $\hat{\mathbb{H}}=\hat{\mathbb{Y}}$ and assume that $B$ is $\hat{y}_{T}$-measurable.

In more detail, we consider one of the following two cases.

Case (I): $B \in L^{\infty}\left(\mathcal{Y}_{T}\right), \lambda$ is $\mathbb{Y}$-predictable, and $\rho$ is $\mathbb{Y}$-predictable.

Case (II): $B \in L^{\infty}\left(\hat{\mathcal{Y}}_{T}\right), \lambda$ is $\mathbb{F}^{S, \hat{Y}}$-predictable, and $\lambda$ is $\mathbb{F}$-predictable.

The assumption in case (II) that $\lambda$ is $\mathbb{F}^{S, \hat{Y}}$-predictable is quite natural since $S$ and $\hat{Y}$ are the quantities observable for our investor. Moreover, it guarantees, by Lemma 1, below, that $\mathbb{F}^{Y, Y^{\perp}} \subseteq \mathbb{F}^{S, \hat{Y}}$, i.e. the two basic driving Brownian motions $Y$ and $Y^{\perp}$ are observable from $S$ and $\hat{Y}$. In particular, if we take $\mathbb{G}=\mathbb{F}^{Y, Y^{\perp}}$, the a priori condition that $\lambda$ is $\mathbb{F}^{S, \hat{Y}}$-predictable turns out to be innocent a posteriori. 
To motivate our model choice, we discuss a typical example for each case.

Consider case (I). Here one should think of a stochastic volatility model, where $\mu$ and $\sigma$ are $\mathbb{Y}$-predictable and the contingent claim $B$ depends only on $\sigma$ (e.g. a variance swap). The stock $S$ is driven by the Brownian motion $W$, whereas its drift and volatility depend on a second factor $Y$. Our approach allows us to consider the situation where the correlation between $W$ and $Y$ is not constant, but more realistically a functional of $Y$.

In this setting $B$ is naturally $\mathcal{Y}_{T}$-measurable and $\lambda=\mu / \sigma$ is $\mathbb{Y}$-predictable like $\mu$ and $\sigma$. The only genuine condition is that $\rho$ should be $\mathbb{Y}$-predictable, which we technically need to guarantee that $\hat{Y}$ has the representation property not only in $\hat{\mathbb{Y}}$, but also in $\mathbb{Y}$.

Consider case (II). A good application here comes from executive stock options. Think of a manager who receives call options on the stock (driven by $\hat{Y}$ ) of her company as part of her performance-related compensation. The manager must not trade the company stock and all its derivatives because of legal restrictions. However, she might be able to trade other, correlated stocks. So $S$ here is a market index, a representative portfolio of other companies in the same line of business, or the stock of a leading company in the same line of business, which serves as a benchmark. We assume that the only source of incompleteness is the fact that the manager is not allowed to directly trade the stock of her company. In particular, we suppose that the market formed by the bank account and $S$ is complete by assuming that $\mu$ and $\sigma$ are both $\mathbb{F}$ and $\mathbb{F}^{S}$-predictable. Then $\mathbb{F}=\mathbb{F}^{S}$, i.e. the uncertainty $(\mathbb{F})$ about $S$ equals the information $\left(\mathbb{F}^{S}\right)$ available from $S$. This follows from (2) because $\sigma$ is bounded away from 0 . We then provide a fair value for the executive options in such a situation.

In this setting $\lambda=\mu / \sigma$ is $\mathbb{F}$ - and $\mathbb{F}^{S}$-predictable like $\mu$ and $\sigma$. The only genuine condition here is that $B$ is $\hat{y}_{T}$-measurable, and the next remark explains why this is natural. Equivalently, that remark clarifies why we view the nontradable asset here as driven by $\hat{Y}$ and not by $Y$.

In both cases the measurability assumptions make precise the underlying idea: the payoff $B$ is driven by $Y$ (or $\hat{Y}$ ), whereas hedging can only be done in $S$ which is imperfectly correlated with $Y$ (or $\hat{Y}$ ). The examples also illustrate two reasons why direct hedging in the stochastic process underlying $B$ may be impossible; either its driver is not traded at all (e.g. a volatility or a consumer price index), or it is traded in principle but not tradable by our investor, due to legal, liquidity, practicability, cost, or other reasons.

Remark. To see why $\hat{y}_{T}$-measurability of $B$ is reasonable in case (II), recall that $B$ is a claim on some asset $Z$, and write $\mathrm{d} Y=\rho \mathrm{d} W+\sqrt{1-\rho^{2}} \mathrm{~d} W^{\perp}$ for a $(\mathbb{G}, \mathrm{P})$-Brownian motion $W^{\perp}$ P-independent of $W$. The asset change $\mathrm{d} Z$ is driven by two factors: the market development $\mathrm{d} S / S$ of the benchmark $S$ and company specific risks $\mathrm{d} W^{\perp}$. To determine the genuine driver of $Z$, we weight the two factors by the correlation process $\rho$, but first make them comparable by 'normalizing' $\mathrm{d} S / S$, which means that we use $(1 / \sigma)(\mathrm{d} S / S)=\mathrm{d} \hat{W}$ instead of $\mathrm{d} S / S$. Thus, $Z$ is driven by

$$
\rho \mathrm{d} \hat{W}+\sqrt{1-\rho^{2}} \mathrm{~d} W^{\perp}=\rho \lambda \mathrm{d} s+\rho \mathrm{d} W+\sqrt{1-\rho^{2}} \mathrm{~d} W^{\perp}=\rho \lambda \mathrm{d} s+\mathrm{d} Y=\mathrm{d} \hat{Y},
$$

using (3). Hence, assuming the $Z$-dependent claim $B$ to be $\hat{y}_{T}$-measurable is more natural than having it $y_{T}$-measurable. Note that the filtrations $\hat{\mathbb{Y}}$ and $\mathbb{Y}$ differ in general, but coincide if $\rho$ and $\lambda$ are deterministic.

Let us now briefly look at the information available to our investor. We always assume that the tradable stock $S$ is observable. In addition, we assume in both cases (I) and (II) that the driver for the uncertainty behind $B$ (i.e. $Y$ or $\hat{Y}$, respectively) is also observable. The following result shows that the observable filtration then contains the filtration $\mathbb{F}^{Y, Y^{\perp}}$ of the underlying 
Brownian motions, and this justifies why we always use $\mathbb{G} \supseteq \mathbb{F}^{Y, Y^{\perp}}$ to describe the information on which our strategies $\pi \in \mathcal{A}_{t}$ must be based.

Lemma 1. In case (I), $\mathbb{F}^{Y, Y^{\perp}} \subseteq \mathbb{F}^{S, Y}$, and in case (II), $\mathbb{F}^{Y, Y^{\perp}} \subseteq \mathbb{F}^{S, \hat{Y}}$.

Proof. Note that the argument in each case uses only the middle condition on $\lambda$. For brevity, we write $Z \in \mathbb{F}^{X}$ to mean that $Z$ is $\mathbb{F}^{X}$-predictable.

Consider case (I). By (2), $\langle S\rangle=\int \sigma^{2} S^{2} \mathrm{~d} s$ and $\langle S\rangle \in \mathbb{F}^{S}$ since it is a continuous pathwise quadratic variation; so $\sigma S=+\sqrt{\sigma^{2} S^{2}} \in \mathbb{F}^{S}$ and, hence, $\sigma \in \mathbb{F}^{S}$ also. Next, $\lambda \in \mathbb{Y}$ by assumption, and so $\mu=\sigma \mid b m \lambda \in \mathbb{F}^{S, Y}$. Because $\sigma$ is bounded away from 0 , we obtain

$$
W=\int \frac{1}{\sigma S} \mathrm{~d} S-\int \frac{\mu}{\sigma} \mathrm{d} s \in \mathbb{F}^{S, Y} .
$$

As a consequence, $\rho \in \mathbb{F}^{S, Y}$, since it is the density of $\langle W, Y\rangle$ with respect to Lebesgue measure and $\langle W, Y\rangle \in \mathbb{F}^{S, Y}$, being a continuous pathwise quadratic covariation. Finally, $|\rho|$ is bounded away from 1; so solving (1) for $Y^{\perp}$ implies that $Y^{\perp} \in \mathbb{F}^{S, Y}$ and, therefore, $\mathbb{F}^{Y, Y^{\perp}} \subseteq \mathbb{F}^{S, Y}$.

Consider case (II). Again, $\sigma \in \mathbb{F}^{S}$. Moreover, (1), (2), and the definition of $\hat{Y}$ given in (3) yield $\langle S, \hat{Y}\rangle_{\hat{Y}}=\int \sigma S \rho \mathrm{d} s$ so that $\rho \in \mathbb{F}^{S, \hat{Y}}$. Because $\lambda \in \mathbb{F}^{S, \hat{Y}}$ by assumption, we obtain $\mu=\sigma \lambda \in \mathbb{F}^{S, \hat{Y}}$, and now we can argue as in case (I) to deduce that $Y^{\perp} \in \mathbb{F}^{S, \hat{Y}}$. Moreover, $Y=\hat{Y}-\int \rho \lambda \mathrm{d} s \in \mathbb{F}^{S, \hat{Y}}$ and, hence, $\mathbb{F}^{Y, Y^{\perp}} \subseteq \mathbb{F}^{S, \hat{Y}}$. This completes the proof.

The following two theorems give explicit formulae for the value $V^{B}$ and the indifference value $b$ in cases (I) and (II). To facilitate comparisons with the literature, we state them for a spot interest rate on the bank account given by a bounded deterministic Borel-measurable function $r:[0, T] \rightarrow[0, \infty)$. Our results and arguments given for $r \equiv 0$ easily extend to this case; allowing $r$ to be stochastic, however, would be a different issue.

Theorem 2. Consider the setting and the assumptions from Subsection 2.1, and recall $\underline{\delta}_{t}$ and $\bar{\delta}_{t}$ from (14). In case (I) define

$$
\hat{B}:=\gamma B-\frac{1}{2} \int_{t}^{T} \frac{\left(\mu_{s}-r(s)\right)^{2}}{\sigma_{s}^{2}} \mathrm{~d} s \quad \text { and } \quad \hat{0}:=-\frac{1}{2} \int_{t}^{T} \frac{\left(\mu_{s}-r(s)\right)^{2}}{\sigma_{s}^{2}} \mathrm{~d} s .
$$

Then there exist $g_{t}$-measurable random variables $\delta_{t}^{\hat{B}}$ and $\delta_{t}^{\hat{0}}$ with values in $\left[\underline{\delta}_{t}, \bar{\delta}_{t}\right]$ such that

$$
V_{t}^{B}\left(x_{t}\right)(\omega)=-\left.\exp \left(-\gamma x_{t}(\omega) \exp \left(\int_{t}^{T} r(s) \mathrm{d} s\right)\right)\left(\mathrm{E}_{\hat{\mathrm{P}}}\left[\exp (\hat{B})^{1 / \delta} \mid y_{t}\right](\omega)\right)^{\delta}\right|_{\delta=\delta_{t}^{\hat{B}}(\omega)}
$$

and

$$
b_{t}(\omega)=\left.\frac{\exp \left(-\int_{t}^{T} r(s) \mathrm{d} s\right)}{\gamma} \log \frac{\left(\mathrm{E}_{\hat{\mathrm{P}}}\left[\exp (\hat{B})^{1 / \delta} \mid y_{t}\right](\omega)\right)^{\delta}}{\left(\mathrm{E}_{\hat{\mathrm{P}}}\left[\exp (\hat{0})^{1 / \delta^{\prime}} \mid \mathcal{y}_{t}\right](\omega)\right)^{\delta^{\prime}}}\right|_{\delta^{\prime}=\delta_{t}^{\hat{0}}(\omega), \delta=\delta_{t}^{\hat{B}}(\omega)}
$$

for almost all $\omega \in \Omega$ and every bounded $g_{t}$-measurable random variable $x_{t}$.

Theorem 3. Consider the setting and the assumptions from Subsection 2.1, and recall $\underline{\delta}_{t}$ and $\bar{\delta}_{t}$ from (14). In case (II) there exists a $g_{t}$-measurable random variable $\delta_{t}^{\gamma B}$ with values in $\left[\underline{\delta}_{t}, \bar{\delta}_{t}\right]$ such that

$$
\begin{aligned}
V_{t}^{B}\left(x_{t}\right)(\omega)=- & \exp \left(-\gamma x_{t}(\omega) \exp \left(\int_{t}^{T} r(s) \mathrm{d} s\right)-\frac{1}{2} \mathrm{E}_{\hat{\mathrm{P}}}\left[\int_{t}^{T} \frac{\left(\mu_{s}-r(s)\right)^{2}}{\sigma_{s}^{2}} \mathrm{~d} s \mid \mathcal{F}_{t}\right]\right) \\
& \times\left.\left(\mathrm{E}_{\hat{\mathrm{P}}}\left[\exp (\gamma B)^{1 / \delta} \mid \hat{y}_{t}\right](\omega)\right)^{\delta}\right|_{\delta=\delta_{t}^{\gamma B}(\omega)}
\end{aligned}
$$


and

$$
b_{t}(\omega)=\left.\frac{\exp \left(-\int_{t}^{T} r(s) \mathrm{d} s\right)}{\gamma} \log \left(\mathrm{E}_{\hat{\mathrm{P}}}\left[\exp (\gamma B)^{1 / \delta} \mid \hat{y}_{t}\right](\omega)\right)^{\delta}\right|_{\delta=\delta_{t}^{\gamma B}(\omega)}
$$

for almost all $\omega \in \Omega$ and every bounded $g_{t}$-measurable random variable $x_{t}$.

To the best of our knowledge, results like Theorems 2 or 3 have not been available in the literature so far; all previous approaches leading to explicit formulae have only considered situations where the correlation $\rho$ is deterministic and constant in time. One nice feature of all the formulae in Theorems 2 and 3 is that the only unknowns are the distortion powers $\delta^{\hat{B}}$, $\delta^{\hat{0}}$, or $\delta^{\gamma B}$, and we have precise bounds for these in terms of bounds on the correlation $\rho$. In general, each such power is random (in a $\mathbb{G}$-adapted way) and depends on $B$ via $\hat{B}$. Since we have assumed that $g_{0}$ is trivial, $\delta_{0}^{\hat{B}}$ is deterministic, but may still depend on $\hat{B}$. However, if the correlation $\rho$ is deterministic and constant in time, the functions $\underline{\delta}$ and $\bar{\delta}$ in (14) coincide and equal $1 /\left(1-\rho^{2}\right)$, and then $\delta^{\hat{B}}=1 /\left(1-\rho^{2}\right)$ becomes constant and independent of $B$ or $\hat{B}$. This explains why the constant correlation case is easier to handle and understand.

We defer the proofs of Theorems 2 and 3 to Subsection 4.3, and first compare our results with the existing literature.

\subsection{Comparison with the literature}

Exponential utility indifference valuation in Brownian settings has been extensively studied, particularly in Markovian models. An overview with a long literature list is provided in [11]. We present here some references and comment first on the different model assumptions and then on the methods and results.

Recall the model in (1) and (2). Henderson [7], [8], Henderson and Hobson [9], [10], and Musiela and Zariphopoulou [18] all worked in a Markovian framework, where $\mu, \sigma, r$, and $\rho$ are all constant. Henderson [7], [8] and Henderson and Hobson [9], [10] used a nontraded asset $Z$ satisfying, for some constants $a>0$ and $b \in \mathbb{R}$,

$$
\frac{\mathrm{d} Z_{s}}{Z_{s}}=b \mathrm{~d} s+a \mathrm{~d} Y_{s}, \quad 0 \leq s \leq T, Z_{0}>0,
$$

and the contingent claim $B=B\left(Z_{T}\right)$ was a function of the terminal value $Z_{T}$ alone. As in (1), $Y$ is a Brownian motion having correlation $\rho$ with $W$. Musiela and Zariphopoulou [18] contains a slightly more general diffusion setting where $a_{s}=a\left(Z_{s}, s\right)$ and $b_{s}=b\left(Z_{s}, s\right)$ may depend on the current level of $Z$ and on time. Monoyios [16] studied a similar model where $\sigma$ and $\lambda=\mu / \sigma$ are not constant, but $\sigma$ equals $Z$ and $\lambda_{s}=\lambda\left(Z_{s}\right)$ is a function of the current level of $Z$. Grasselli and Hurd [6] and Stoikov and Zariphopoulou [20] considered claims which depend not only on $Z_{T}$, but also in a certain way on the trajectory of $Z$. In contrast to all the above Markovian models, Tehranchi [21] analyzed a more general situation very similar to case (I); but his approach is still restricted to a constant correlation $\rho$.

To the best of our knowledge, the only article where $\rho$ is not constant is by Benth and Karlsen [2], who studied a Markovian setting with $\rho=\rho\left(Z_{S}\right)$ depending on the present level of the nontraded asset $Z$. They showed that the minimal entropy martingale measure can be expressed in terms of the solution of a semilinear PDE for which they proved existence and uniqueness of a classical solution. However, they had no claim $B$ and they also did not derive any general explicit formulae.

Remark. All the Markovian models above with constant $\mu, \sigma, r, \rho, a$, and $b$ satisfy the measurability conditions for both cases (I) and (II). It is therefore somewhat arbitrary whether 
one views them as stochastic volatility or rather as executive stock option models. (Indeed, only our general model makes this precise distinction really possible.) The subsequent generalizations in [6], [16], [20], and [21] all head towards our case (I), whereas models from case (II) have not yet been studied for nondeterministic $\lambda$ or $\rho$. In that sense, it seems fair to say that our formulation with a clear distinction between cases (I) and (II) represents a significant generalization of previously considered models.

We now recall and comment on how explicit formulae for the indifference value $b$ are derived in the literature. As in Subsection 2.1, one usually first derives an expression for the value $V^{B}$ and then obtain a formula for $b$ via (7). In a Markovian model the usual approach is to condition on the current state of the nontraded asset $Z$ in (32), i.e. to write

$$
V_{t}^{B}\left(x_{t}\right)=v\left(x_{t}, z_{t}, t\right):=\underset{\pi \in \mathcal{A}_{t}}{\operatorname{ess} \sup } \mathrm{E}\left[U\left(X_{T}^{x_{t}, \pi}-B\left(Z_{T}\right)\right) \mid X_{t}^{x_{t}, \pi}=x_{t}, Z_{t}=z_{t}\right] .
$$

Henderson [7], Henderson and Hobson [9], [10], and later Musiela and Zariphopoulou [18] first wrote the Hamilton-Jacobi-Bellman nonlinear PDE for the value function $v$. Exploiting the scaling properties of the exponential utility function $U$, they tried an ansatz of the form

$$
v(x, z, t)=U(x) F(z, t),
$$

which results in a nonlinear PDE for $F$. A clever power transformation,

$$
F(z, t)=f(z, t)^{1 /\left(1-\rho^{2}\right)},
$$

reduces this to a linear and solvable PDE for $f$. This yields an explicit formula for $v$ and, thus, also for $b$ via (7).

The idea to convert a nonlinear PDE into a linear PDE by a power transformation was introduced in [22] for optimal portfolio management problems with nontraded assets when the utility is of the separable constant relative risk aversion type: the payoff $B\left(Z_{T}\right)$ of the claim is multiplied by a power of the investor's final portfolio value $X_{T}^{x_{t}, \pi}$, i.e. is of the form $\tilde{U}\left(X_{T}^{x_{t}, \pi}, B\left(Z_{T}\right)\right)=B\left(Z_{T}\right)\left|X_{T}^{x_{t}, \pi}\right|^{\gamma} / \gamma$ with $0 \neq \gamma<1$. The application of the power transformation, (33), to exponential utility indifference valuation appeared first in [7], [9], and [10], and later in [18]. The exponent $\delta:=1 /\left(1-\rho^{2}\right)$ from (33) is called the distortion power, a terminology due to Zariphopoulou [22], and the approach is also known as the distortion method. Henderson [7] and Henderson and Hobson [9], [10] also derived an approximation (for a small number of claims) of the power utility indifference value, which they compared with the exponential indifference value. Henderson [8] examined the latter criterion and incentives for executive stock options in the Markovian model of [7], [9], and [10]. Monoyios [16] derived a representation of the optimal measure for the dual problem by combining the distortion method with general duality results. He further considered the optimization problem under power utility, but without random endowment. Grasselli and Hurd [6] and Stoikov and Zariphopoulou [20] presented explicit formulae for the exponential utility indifference value of a path-dependent claim on the volatility. But, as already mentioned, all these approaches work only in a Markovian model and if the instantaneous correlation $\rho$ between $W$ and $Y$ is constant.

In an alternative approach, Tehranchi [21] obtained an explicit expression for $V_{t}^{B}\left(x_{t}\right)$ in (6) with $t=0$. He first proved a Hölder-type inequality which he then applied to determine $V_{0}^{B}\left(x_{0}\right)$, and this also yielded an explicit formula for the indifference value at time 0 . Tehranchi's method has the advantage that it needs no Markovian assumption and can treat general (bounded) $y_{T}$-measurable claims; but this method is still restricted to situations with constant correlation. 
The distortion power $\delta=1 /\left(1-\rho^{2}\right)$ from (33) arises there as an exponent in the Hölder-type inequality.

In all the above approaches $\delta$ plays an important role, and it is crucial that it is deterministic and constant in time. We also use a power transformation in (17) with a power $\delta$ which must be constant, whereas $\delta=1 /\left(1-\rho^{2}\right)$ in the above methods depends on $\rho$. This explains why we use two different powers in our proof of Theorem 1: $\bar{\delta}_{t}$ gives an upper bound for $\hat{V}_{t}^{\hat{B}}$ in Proposition 1 and $\underline{\delta}_{t}$ gives a lower bound for $\hat{V}_{t}^{\hat{B}}$ in Proposition 2. The deeper reason why we can deal with a random correlation $\rho$ is then a monotonicity property, as will be explained in Section 5 .

Remarks. 1. In Theorem 2 we can replace $\hat{\mathrm{P}}$ by the restriction $\mathrm{Q}$ of $\hat{\mathrm{P}}$ to $y_{T}$, because $\hat{B}$ and $\hat{0}$ are $y_{T}$-measurable; so, for almost all $\omega \in \Omega$, we have

$$
b_{t}(\omega)=\left.\frac{\exp \left(-\int_{t}^{T} r(s) \mathrm{d} s\right)}{\gamma} \log \frac{\left(\mathrm{E}_{\mathrm{Q}}\left[\exp (\hat{B})^{1 / \delta} \mid y_{t}\right](\omega)\right)^{\delta}}{\left(\mathrm{E}_{\mathrm{Q}}\left[\exp (\hat{0})^{1 / \delta^{\prime}} \mid y_{t}\right](\omega)\right)^{\delta^{\prime}}}\right|_{\delta^{\prime}=\delta_{t}^{\hat{0}}(\omega), \delta=\delta_{t}^{\hat{B}}(\omega)} .
$$

Since $\rho$ and $\lambda$ are $\mathbb{Y}$-predictable in case (I), (1) and (4) explicitly yield

$$
\frac{\mathrm{dQ}}{\mathrm{dP}}:=\mathrm{E}_{\mathrm{P}}\left[\frac{\mathrm{d} \hat{\mathrm{P}}}{\mathrm{dP}} \mid y_{T}\right]=\mathscr{E}\left(-\int \rho \lambda \mathrm{d} Y\right)_{0, T} .
$$

This formula is used by Tehranchi [21] to define Q in his setting with constant $\rho$. Similarly, in Theorem 3 we could replace $\hat{\mathrm{P}}$ by the restriction $\hat{\mathrm{Q}}$ of $\hat{\mathrm{P}}$ to $\hat{y}_{T}$. However, this is less useful because $\hat{Q}$, unlike $\mathrm{Q}$, has in general no explicit form.

2. Apart from exponential utility, Tehranchi [21] also explicitly determined $V_{0}^{B}$ for constant $\rho$ when the investor's utility was of the same separable form as in [22], i.e. of the form $\tilde{U}\left(X_{T}^{x_{t}, \pi}, B\right)=B\left|X_{T}^{x_{t}, \pi}\right|^{\gamma} / \gamma$ with $0 \neq \gamma<1$, or $\tilde{U}\left(X_{T}^{x_{t}, \pi}, B\right)=B \log X_{T}^{x_{t}, \pi}$. These results could be extended with our techniques, as for exponential utility, to all times $t$ and to random $\rho$. But we give no details since this provides no essential new insights and, above all, does not help in finding an indifference value, because the above utilities are not of the form $U\left(X_{T}^{x_{t}, \pi}+B\right)$ required for a natural formulation.

3. The original motivation for this paper was that we were intrigued by the elegantly simple and yet general approach of Tehranchi [21]. Along the way, we then discovered that not all arguments in [21] seem completely rigorous; the proof there of Lemma 4.2 is not quite clear (measurability of integrands?), and we see no argument why the portfolios constructed in Propositions 3.3-3.5 satisfy the integrability requirements to lie in the respective classes $\mathcal{A}$ of admissible strategies. Moreover, the proofs of these propositions also contain an incorrect statement; in general, a Brownian motion $W$ and a process of the form $W+\int \lambda \mathrm{d} s$ do not generate the same filtration or $\sigma$-field, even if $\lambda$ is predictable with respect to the filtration generated by $W$. A counterexample is given in [4]. Despite all this, the final results in [21] are essentially correct; one way to circumvent the last problem is contained in the proof of our Theorem 2.

\subsection{Proofs of Theorems 2 and 3}

We first need the following general result which says that the class $(D)$ property behaves, under a change to an equivalent probability measure, in the same way as martingales. This is very intuitive and probably folklore, but we have not found it anywhere. 
Lemma 2. Denote by $Z^{\prime}$ the $\mathrm{P}$-density process of a probability measure $\mathrm{P}^{\prime}$ equivalent to $\mathrm{P}$, i.e. $Z_{s}^{\prime}:=\mathrm{E}_{\mathrm{P}}\left[\mathrm{dP}^{\prime} / \mathrm{dP} \mid g_{s}\right], s \in[0, T] . A \mathbb{G}$-adapted càdlàg process $\Lambda$ is of $\mathrm{P}^{\prime}$-class $(D)$ if and only if $\Lambda Z^{\prime}$ is of $\mathrm{P}$-class $(D)$.

Proof. By symmetry and Bayes's formula, it is enough to prove the 'only if' part. Take a $\mathbb{G}$-adapted càdlàg process $\Lambda$ of $\mathrm{P}^{\prime}$-class $(D)$, and fix $\varepsilon>0$. We want to find $K>0$ with $\sup _{\tau} \mathrm{E}_{\mathrm{P}}\left[\left|\Lambda_{\tau}\right| Z_{\tau}^{\prime} \mathbf{1}_{\left\{\left|\Lambda_{\tau}\right| Z_{\tau}^{\prime}>K\right\}}\right] \leq \varepsilon$, where the supremum is taken over all $\mathbb{G}$-stopping times $\tau$. Using the fact that $\mathrm{dP}^{\prime}=Z_{\tau}^{\prime} \mathrm{dP}$ on $g_{\tau}$ gives

$$
\mathrm{E}_{\mathrm{P}}\left[\left|\Lambda_{\tau}\right| Z_{\tau}^{\prime} \mathbf{1}_{\left\{\left|\Lambda_{\tau}\right| Z_{\tau}^{\prime}>K\right\}}\right]=\mathrm{E}_{\mathrm{P}^{\prime}}\left[\left|\Lambda_{\tau}\right| \mathbf{1}_{\left\{\left|\Lambda_{\tau}\right| Z_{\tau}^{\prime}>K\right\}}\right]
$$

Since $\Lambda$ is of $\mathrm{P}^{\prime}$-class $(D), m:=1 \vee \sup _{\tau} \mathrm{E}_{\mathrm{P}^{\prime}}\left[\left|\Lambda_{\tau}\right|\right]$ is finite and there exists $d_{1}>0$, which does not depend on $\tau$, such that

$$
A \in g_{T} \text { with } \mathrm{P}^{\prime}[A] \leq d_{1} \text { and } \tau \text { a } \mathbb{G} \text {-stopping time } \quad \Longrightarrow \quad \mathrm{E}_{\mathrm{P}^{\prime}}\left[\left|\Lambda_{\tau}\right| \mathbf{1}_{A}\right] \leq \varepsilon
$$

Because $\mathrm{P}^{\prime} \ll \mathrm{P}$ by assumption, there exists $d_{2}>0$ such that

$$
A \in g_{T} \text { with } \mathrm{P}[A] \leq d_{2} \quad \Longrightarrow \quad \mathrm{P}^{\prime}[A] \leq d_{1}
$$

Set $K:=m / d_{2}$ and use Markov's inequality to obtain

$$
\mathrm{P}\left[\left|\Lambda_{\tau}\right| Z_{\tau}^{\prime}>K\right] \leq \frac{1}{K} \mathrm{E}_{\mathrm{P}}\left[\left|\Lambda_{\tau}\right| Z_{\tau}^{\prime}\right]=\frac{1}{K} \mathrm{E}_{\mathrm{P}^{\prime}}\left[\left|\Lambda_{\tau}\right|\right] \leq \frac{m}{K}=d_{2}
$$

for any $\mathbb{G}$-stopping time $\tau$. Now (35) and (36) yield $\mathrm{E}_{\mathrm{P}^{\prime}}\left[\left|\Lambda_{\tau}\right| \mathbf{1}_{\left\{\left|\Lambda_{\tau}\right| Z_{\tau}^{\prime}>K\right\}}\right] \leq \varepsilon$ uniformly over $\tau$. This completes the proof.

Now we can prove Theorems 2 and 3 by applying Theorem 1 .

Proof of Theorem 2. Equation (31) follows directly from (7) and (30). To prove (30), we apply Theorem 1 with $\hat{B}:=\bar{B}=\gamma B-\frac{1}{2} \int_{t}^{T} \lambda_{s}^{2} \mathrm{~d} s$ and $\hat{\mathbb{H}}:=\mathbb{Y}$. Comparing (13) with (11) shows that it only remains to argue that

(i) $\hat{Y}$ has the representation property in $\mathbb{Y}$, and

(ii) $\pi \in \mathcal{A}_{t}$ is equivalent to $\gamma \pi \sigma-\lambda \in \hat{\mathcal{A}}_{t}$.

The latter property follows directly from Lemma 1 which yields the fact that $\exp \left(-\int \gamma \pi \sigma \mathrm{d} \hat{W}\right)$ is of P-class $(D)$ if and only if $\exp \left(-\int(\gamma \pi \sigma-\lambda) \mathrm{d} \hat{W}\right)$ is of $\hat{\mathrm{P}}$-class $(D)$, because $\int \lambda^{2} \mathrm{~d} s$ is bounded. Property (i) is deduced from Itô's representation theorem in the form of Lemma 1.6.7 of [13]. In more detail, consider the restriction $\mathrm{Q}$ of $\hat{\mathrm{P}}$ to $\mathcal{y}_{T}$, given as in (34) by

$$
\frac{\mathrm{dQ}}{\mathrm{dP}}=\mathrm{E}_{\mathrm{P}}\left[\frac{\mathrm{d} \hat{\mathrm{P}}}{\mathrm{dP}} \mid y_{T}\right]=\mathcal{E}\left(-\int \rho \mid b m \lambda \mathrm{d} Y\right)_{0, T},
$$

because $\lambda$ and $\rho$ are $\mathbb{Y}$-predictable. Note that this uses the assumptions of case (I). Here $\hat{Y}$ is also a ( $\mathbb{Y}, Q)$-Brownian motion, and Lemma 1.6.7 of [13] now yields the fact that any ( $\mathbb{Y}, Q)$ martingale $L$ is of the form $L=L_{0}+\int \zeta \mathrm{d} \hat{Y}$ for a $\mathbb{Y}$-predictable $\zeta$ with $\int_{0}^{T} \zeta_{s}^{2} \mathrm{~d} s<\infty$ a.s. This crucially needs the fact that $\rho \lambda$ is $\mathbb{Y}$-predictable, to ensure that $\hat{Y}=Y+\int \rho \lambda \mathrm{d} s$ from (3) is $\mathbb{Y}$-adapted. 
Proof of Theorem 3. As in the proof of Theorem 2, we apply Theorem 1, but now with $\hat{B}:=\gamma B$ and $\hat{\mathbb{H}}:=\hat{\mathbb{Y}}$. Of course, the $(\hat{\mathbb{H}}, \hat{\mathrm{P}})$-Brownian motion $\hat{Y}$ then has the representation property in $\hat{\mathbb{H}}$. To get rid of the term $\frac{1}{2} \int_{t}^{T} \lambda_{s}^{2} \mathrm{~d} s$ in $\bar{B}$ in (11), we again use Itô's representation theorem, as in Lemma 1.6.7 of [13], and obtain an $\mathbb{F}$-predictable process $\eta=\left(\eta_{s}\right)_{t \leq s \leq T}$ with

$$
\frac{1}{2} \int_{t}^{T} \lambda_{s}^{2} \mathrm{~d} s=\frac{1}{2} \mathrm{E}_{\hat{\mathrm{P}}}\left[\int_{t}^{T} \lambda_{s}^{2} \mathrm{~d} s \mid \mathcal{F}_{t}\right]+\int_{t}^{T} \eta_{s} \mathrm{~d} \hat{W}_{s} \quad \text { and } \quad \mathrm{E}_{\hat{\mathrm{P}}}\left[\int_{t}^{T} \eta_{s}^{2} \mathrm{~d} s\right]<\infty .
$$

Here we use the fact that $\lambda$ is $\mathbb{F}$-predictable in case (II), where we recall that $\mathbb{F}=\mathbb{F}^{W}$. Finally, a comparison of (11) and (13) with $\hat{B}=\gamma B$ shows that it remains to prove that $\pi \in \mathcal{A}_{t}$ if and only if $\gamma \pi \sigma-\lambda+\eta \in \hat{\mathcal{A}}_{t}$. But this follows as in the proof of Theorem 2 from Lemma 1, using the fact that $\int \eta \mathrm{d} \hat{W}$ is, like $\int \lambda^{2} \mathrm{~d} s$, uniformly bounded.

\section{On the monotonicity in the correlation}

In this section we explain both intuitively and mathematically why we can obtain results even for a random correlation $\rho$.

For a constant correlation $\rho$, the abstract optimization problem, (13), has, by Theorem 1 (or from Tehranchi [21] for $t=0)$, an explicit value, namely, (15) with $\delta=1 /\left(1-\rho^{2}\right)$. This expression is continuous in $\rho$ and increasing in $|\rho|$ for fixed $\hat{\mathrm{P}}$, and the intuition is as follows. The endowment $\hat{B}$ is driven by $\hat{Y}$, whereas hedging can only be done in $\hat{W}$, which is imperfectly correlated with $\hat{Y}$. If the correlation between $\hat{W}$ and $\hat{Y}$ is increased, better hedging is possible; so the value of the optimization problem, (13), decreases. (Note that (13) gives us minus the maximal expected utility.)

If we can extend the above monotonicity to a general correlation, it is clear why we can obtain the explicit structure in Theorem 1. Indeed, if $\rho$ is random but lies between two bounds, the corresponding optimization problem must, by monotonicity, have an explicit expression with the same basic structure-and of course the interpolating distortion power may now be random and depend on $\hat{B}$.

Let us now introduce more precise notation by writing (13) as

$$
\hat{V}_{t}^{\hat{B}}\left(\rho^{\prime}, \hat{\mathrm{P}}\right):=-\underset{\hat{\pi} \in \hat{\mathcal{A}}_{t}\left(\rho^{\prime}\right)}{\operatorname{ess} \operatorname{Enf}_{\hat{\mathrm{P}}}}\left[\exp \left(-\int_{t}^{T} \hat{\pi}_{s} \mathrm{~d} \hat{W}_{s}\left(\rho^{\prime}\right)+\hat{B}\right) \mid g_{t}\right]
$$

for a $\mathbb{G}$-predictable process $\rho^{\prime}$ denoting the instantaneous correlation between the $(\mathbb{G}, \hat{\mathrm{P}})$ Brownian motions $\hat{W}\left(\rho^{\prime}\right)$ and $\hat{Y}$; the set $\hat{\mathcal{A}}_{t}\left(\rho^{\prime}\right)$ depends on $\rho^{\prime}$ through the $\hat{\mathrm{P}}$-class $(D)$ condition on $\left(\exp \left(-\int_{t}^{s} \hat{\pi}_{y} \mathrm{~d} \hat{W}_{y}\left(\rho^{\prime}\right)\right)\right)_{t \leq s \leq T}$. Note that if we change $\rho^{\prime}$, only $\hat{W}\left(\rho^{\prime}\right)$ and all the expressions depending on it will change. This is reasonable; clearly, $\hat{B}$ and $\hat{\mathbb{H}}$ should not be affected.

The above intuitive argument now says that if we keep $\hat{\mathrm{P}}$ fixed and vary $\rho^{\prime}$, we obtain a monotonicity, which is made precise in the following result.

Proposition 3. Let $\hat{\mathrm{P}}$ be fixed, and suppose that $\rho^{\prime}$ and $\rho^{\prime \prime}$ are $\mathbb{G}$-predictable processes such that $\left|\rho^{\prime}\right| \leq c_{1} \leq\left|\rho^{\prime \prime}\right| \leq c_{2}<1$ on $[t, T] \times \Omega$ for some constants $c_{1}$ and $c_{2}$. Then

$$
\hat{V}_{t}^{\hat{B}}\left(\rho^{\prime}, \hat{\mathrm{P}}\right) \leq \hat{V}_{t}^{\hat{B}}\left(\rho^{\prime \prime}, \hat{\mathrm{P}}\right) \text { a.s. }
$$

Proof. This follows from applying Theorem 1 twice, once for $\hat{V}_{t}^{\hat{B}}\left(\rho^{\prime}, \hat{\mathrm{P}}\right)$ and once for $\hat{V}_{t}^{\hat{B}}\left(\rho^{\prime \prime}, \hat{\mathrm{P}}\right)$, and then using Jensen's inequality.

Remark. Proposition 3 says that $\rho^{\prime} \mapsto \hat{V}_{t}^{\hat{B}}\left(\rho^{\prime}, \hat{\mathrm{P}}\right)$ is monotonic for correlation processes $\rho^{\prime}$ and $\rho^{\prime \prime}$ that can be separated by a constant uniformly in $s$ and $\omega$. We do not know if the weaker assumption $\left|\rho^{\prime}\right| \leq\left|\rho^{\prime \prime}\right|$ on $[t, T] \times \Omega$ is also sufficient to prove the same conclusion. 
The above intuition and Proposition 3 make it tempting to think that the value $V_{t}^{B}(0)$ in (9) is also monotonic in $|\rho|$. However, this is not true in general; we give a counterexample in the next paragraph. The crucial point is that $\hat{\mathrm{P}}$ itself depends on $\rho$ because $W$ does; this can be seen from (1) and (4). So the abstract optimization problem, (13), has the structure $-\left.\hat{V}_{t}^{\hat{B}}\left(\rho^{\prime}, \hat{\mathrm{P}}(\rho)\right)\right|_{\rho^{\prime}=\rho}$, and proving as in Proposition 3 that $\rho^{\prime} \mapsto \hat{V}_{t}^{\hat{B}}\left(\rho^{\prime}, \hat{\mathrm{P}}(\rho)\right)$ is monotonic for fixed $\rho$ need not imply that $\rho \mapsto \hat{V}_{t}^{\hat{B}}(\rho, \hat{\mathrm{P}}(\rho))$ is monotonic.

We now show, by a counterexample, that $\rho \mapsto \hat{V}_{t}^{\hat{B}}(\rho, \hat{\mathrm{P}}(\rho))$ and, thus,

$$
\rho \mapsto V_{t}^{B}(0 ; \rho):=V_{t}^{B}(0)
$$

from (9) are indeed not monotonic in general. In view of Proposition 3, this can only fail in the nonmartingale case, $\lambda \neq 0$, since otherwise $\hat{\mathrm{P}}(\rho)=\mathrm{P}$ does not depend on $\rho$. We take $\rho$ and $\lambda$ both constant, $t=0$, and set $\hat{\mathbb{H}}=\mathbb{Y}$ as in case (I). Then Theorem 1 implies that

$$
\begin{aligned}
\hat{V}_{0}^{\hat{B}}(\rho, \hat{\mathrm{P}}(\rho)) & =-\left(\mathrm{E}_{\hat{\mathrm{P}}(\rho)}\left[\exp (\hat{B})^{1-\rho^{2}}\right]\right)^{1 /\left(1-\rho^{2}\right)} \\
& =-\left(\mathrm{E}_{\mathrm{P}}\left[\exp \left(\hat{B}\left(1-\rho^{2}\right)-\lambda \rho Y_{T}-\frac{T \lambda^{2} \rho^{2}}{2}\right)\right]\right)^{1 /\left(1-\rho^{2}\right)},
\end{aligned}
$$

where we have conditioned on $y_{T}$ under $\mathrm{P}$ and used the fact that $\hat{B}$ is $y_{T}$-measurable. For $\hat{B}=-Y^{n}:=\left(\left(-Y_{T}\right) \wedge n\right) \vee(-n), n \in \mathbb{N}$, dominated convergence and an easy calculation yield

$$
\begin{aligned}
\lim _{n \rightarrow \infty} \hat{V}_{0}^{-Y^{n}}(\rho, \hat{\mathrm{P}}(\rho)) & =-\left(\mathrm{E}_{\mathrm{P}}\left[\exp \left(-Y_{T}\left(1-\rho^{2}\right)-\lambda \rho Y_{T}-\frac{T \lambda^{2} \rho^{2}}{2}\right)\right]\right)^{1 /\left(1-\rho^{2}\right)} \\
& =-\exp \left(\left(-\rho^{2}+2 \lambda \rho+1\right) \frac{T}{2}\right) \\
& =: g(\rho)
\end{aligned}
$$

The mapping $\rho \mapsto g(\rho)$ is clearly not monotonic in $|\rho|$ except in the martingale case, $\lambda=0$. Because of (37), the mapping $\rho \mapsto \hat{V}_{0}^{-Y^{n}}(\rho, \hat{\mathrm{P}}(\rho))$ for big enough $n$ is not monotonic in $|\rho|$ either. If we now consider case (I) with $\gamma=1$ and $B=-Y^{n}$, the proof of Theorem 2 implies that

$$
V_{0}^{-Y^{n}}(0 ; \rho):=V_{0}^{-Y^{n}}(0)=\exp \left(-\frac{\lambda^{2} T}{2}\right) \hat{V}_{0}^{-Y^{n}}(\rho, \hat{\mathrm{P}}(\rho)),
$$

so that $\rho \mapsto V_{0}^{-Y^{n}}(0 ; \rho)$ for big enough $n$ is not monotonic in $|\rho|$ either. This completes our counterexample.

Remark. We can directly show that $\hat{V}_{0}^{-Y_{T}}(\rho, \hat{\mathrm{P}}(\rho))=g(\rho)$ if we adapt the definition of $\hat{\mathcal{A}}_{t}(\rho)$. For such an unbounded $\hat{B}$, we stipulate that $\left(\exp \left(-\int_{t}^{s} \hat{\pi}_{y} \mathrm{~d} \hat{W}_{y}(\rho)+\hat{B}\right)\right)_{t \leq s \leq T}$ instead of $\left(\exp \left(-\int_{t}^{s} \hat{\pi}_{y} \mathrm{~d} \hat{W}_{y}(\rho)\right)\right)_{t \leq s \leq T}$ is of $\hat{\mathrm{P}}$-class $(D)$. The point is then that we can, for this example, explicitly determine $L=\left(\rho^{2}-1\right)(Y+\lambda \rho s)$ defined in (17), and $L$ is obviously a $B M O(\mathbb{G}, \hat{\mathrm{P}}(\rho))$-martingale.

The above counterexample shows that $V^{B}$ is in general not monotonic in $|\rho|$. We now explain the intuition for this. In the martingale case, $\lambda=0$, the value

$$
V_{0}^{-Y_{T}}(0 ; \rho)=\hat{V}_{0}^{-Y_{T}}(\rho, \hat{\mathrm{P}}(\rho))=-\exp \left(\frac{\left(1-\rho^{2}\right) T}{2}\right)
$$


is clearly monotonic in $|\rho|$, and we have already seen why: higher correlation permits better hedging, and so the investor runs less risk and has a higher expected utility. For the semimartingale case, $\lambda \neq 0$, this effect is still there, but now it also interacts with the correlation. Consider, for instance, the case where $\lambda>0$ and $\rho>0$. The optimal strategy $\hat{\pi}^{\star}$ for $\hat{V}_{0}^{0}(\rho, \hat{\mathrm{P}}(\rho))$ is 0 and, hence, the optimal strategy for $V_{0}^{0}(0 ; \rho)$ is $\pi^{\star}=\lambda / \gamma \sigma$; compare the proof of Theorem 2 . This strategy $\pi^{\star}$ makes a positive investment in the stock $S$. Adding $-B=Y_{T}$ leads to a total position with a higher risk, since the correlation $\rho$ between $Y$ and $S$ is positive. To counteract this exposure, the investor will reduce his position in $S$ and smooth out his terminal wealth. Hence, he accepts on average a lower return on his portfolio in $S$ to reduce the risk of his total position. So an increase in correlation yields a higher risk exposure for a fixed strategy; this is compensated for by more conservative (smaller) investment in $S$, leading to a lower return and, hence, a decrease of the value $V_{0}^{-Y_{T}}(0 ; \rho)$. In total, $\rho \mapsto V_{0}^{-Y_{T}}(0 ; \rho)$ can therefore become decreasing in $|\rho|$-despite the better hedging possibility. The above argument explains why this can happen, and (37) shows that it does happen for $0<\rho<\lambda$.

Remark. In a Markovian framework with constant $\rho$ and $\lambda$ the result of Proposition 3 has already been established by Henderson [8] who showed that the indifference value $b$ (or, equivalently in that setting, $\left.V^{B}\right)$ is increasing in $|\rho|$. Henderson's analysis at first sight seems to contradict our nonmonotonic counterexample, and closer inspection reveals that it crucially depends on fixing some parameter called $\delta$ in [8] while varying $\rho$. But this exactly corresponds to our fixing $\hat{P}$ in Proposition 3 while varying $\rho$, and it has in both cases a very natural financial interpretation. In fact, the standard viewpoint in financial theory is that the instantaneous Sharpe ratio $a / b$ of the nontraded asset $Z$ in (32) is not fixed exogenously, but related to $\lambda$ via the correlation $\rho$. This tacit assumption is usually not spelt out explicitly in the finance literature, and the point of our counterexample is to illustrate that monotonicity may fail in its absence.

\section{The multidimensional case}

In this section we extend our main results to the case of more than two Brownian motions. Since most arguments are straightforward generalizations, we only sketch the main differences.

The probabilistic framework consists of an $n$-dimensional $(\mathbb{G}, \mathrm{P})$-Brownian motion $\boldsymbol{Y}$ and an $m$-dimensional $(\mathbb{G}, \mathrm{P})$-Brownian motion $\boldsymbol{W}$, each having P-independent components. Instantaneous correlations are now given by a matrix $\boldsymbol{R}=\left(\rho^{i j}\right)_{i=1, \ldots, n, j=1, \ldots, m}$, where $\rho_{s}^{i j}:=\mathrm{d}\left\langle Y^{i}, W^{j}\right\rangle_{s} / \mathrm{d} s$, and we choose $\boldsymbol{R}$ to be $\mathbb{G}$-predictable. It can be shown that the symmetric positive semidefinite matrix $\boldsymbol{R} \boldsymbol{R}^{\top}$ has nonnegative eigenvalues which are all at most 1. We assume that all the eigenvalues are bounded away from 1 uniformly on $\Omega \times[t, T]$, i.e.

there exists $c<1$ such that spectral radius $\left(\boldsymbol{R} \boldsymbol{R}^{\top}\right) \leq c$ almost everywhere on $\Omega \times[t, T]$.

Recall that $t \in[0, T]$ is fixed. There are $m$ traded risky assets $S=\left(S_{S}^{j}\right)_{j=1, \ldots, m}$ with dynamics

$$
\mathrm{d} S_{s}^{j}=S_{s}^{j} \mu_{s}^{j} \mathrm{~d} s+\sum_{k=1}^{m} S_{s}^{j} \sigma_{s}^{j k} \mathrm{~d} W_{s}^{k}, \quad 0 \leq s \leq T, S_{0}^{j}>0, j=1, \ldots, m ;
$$

the drift vector $\boldsymbol{\mu}=\left(\mu_{s}^{j}\right)_{j=1, \ldots, m}$ and the volatility matrix $\boldsymbol{\sigma}=\left(\sigma_{s}^{j k}\right)_{j, k=1, \ldots, m}$ are $\mathbb{G}$-predictable. We assume that $\sigma$ is invertible, $\lambda:=\sigma^{-1} \boldsymbol{\mu}$ is bounded uniformly (in $s$ and $\omega$ ) and that there exists a constant $C$ such that

$$
C \boldsymbol{\beta}^{\top} \boldsymbol{\beta} \geq \boldsymbol{\beta}^{\top} \boldsymbol{\sigma} \boldsymbol{\sigma}^{\top} \boldsymbol{\beta} \geq \frac{1}{C} \boldsymbol{\beta}^{\top} \boldsymbol{\beta} \quad \text { on } \Omega \times[0, T] \text { for all } \boldsymbol{\beta} \in \mathbb{R}^{m} .
$$


(In other words, $\sigma$ is uniformly both bounded and elliptic.) The processes

$$
\hat{\boldsymbol{W}}:=\boldsymbol{W}+\int \lambda \mathrm{d} s \quad \text { and } \quad \hat{\boldsymbol{Y}}:=\boldsymbol{Y}+\int \boldsymbol{R} \boldsymbol{\lambda} \mathrm{d} s
$$

are Brownian motions under the minimal martingale measure $\hat{\mathrm{P}}$ given by

$$
\frac{\mathrm{d} \hat{\mathrm{P}}}{\mathrm{dP}}:=\varepsilon\left(-\int \lambda \mathrm{d} \boldsymbol{W}\right)_{0, T} .
$$

All other definitions and model assumptions of Subsections 2.1 and 3.1 can be easily translated to this setting and we do not detail this. The multidimensional version of Theorem 1 then reads as follows.

Theorem 4. Under the above assumptions, let $\min \operatorname{EV}\left(\boldsymbol{R}_{s} \boldsymbol{R}_{s}^{\top}\right)$ and $\max \operatorname{EV}\left(\boldsymbol{R}_{s} \boldsymbol{R}_{s}^{\top}\right)$, which is equal to spectral radius $\left(\boldsymbol{R}_{s} \boldsymbol{R}_{s}^{\top}\right)$, denote the smallest and largest eigenvalues of $\boldsymbol{R}_{s} \boldsymbol{R}_{s}^{\top}$, respectively, and define $\underline{\delta}_{t}$ and $\bar{\delta}_{t}$ by

$$
\underline{\delta}_{t}:=\inf _{s \in[t, T]} \frac{1}{\left\|1-\min \operatorname{EV}\left(\boldsymbol{R}_{S} \boldsymbol{R}_{s}^{\top}\right)\right\|_{L^{\infty}}}, \quad \bar{\delta}_{t}:=\sup _{s \in[t, T]}\left\|\frac{1}{1-\max \operatorname{EV}\left(\boldsymbol{R}_{S} \boldsymbol{R}_{s}^{\top}\right)}\right\|_{L^{\infty}} .
$$

Then there exists a $g_{t}$-measurable random variable $\delta_{t}^{\hat{B}}$ with values in $\left[\underline{\delta}_{t}, \bar{\delta}_{t}\right]$ such that

$$
-\hat{V}_{t}^{\hat{B}}(\omega)=\left.\left(\mathrm{E}_{\hat{\mathrm{P}}}\left[\exp (\hat{B})^{1 / \delta} \mid \hat{\mathscr{H}}_{t}\right](\omega)\right)^{\delta}\right|_{\delta=\delta_{t}^{\hat{B}}(\omega)}
$$

for almost all $\omega \in \Omega$.

Outline of the proof. This goes similarly to Theorem 1 via analogues of Propositions 1 and 2, and we only point out where significant changes occur. The analogue to (20) is, for $t \leq s \leq T$,

$$
Z_{s}^{(\hat{\boldsymbol{\pi}})}:=c_{t}^{\delta} M_{t, s}^{(\hat{\boldsymbol{\pi}})} \exp \left(\frac{1}{2} \int_{t}^{s}\left(\left|\hat{\boldsymbol{\pi}}_{y}-\delta \boldsymbol{R}_{y}^{\top} \zeta_{y}\right|^{2}+\delta \zeta_{y}^{\top}\left(\delta\left(\boldsymbol{I}-\boldsymbol{R}_{y} \boldsymbol{R}_{y}^{\top}\right)-\boldsymbol{I}\right) \zeta_{y}\right) \mathrm{d} y\right)
$$

with $M^{(\hat{\boldsymbol{\pi}})}:=\mathcal{E}\left(\int \delta \zeta \mathrm{d} \hat{\boldsymbol{Y}}-\int \hat{\boldsymbol{\pi}} \mathrm{d} \hat{\boldsymbol{W}}\right)$ as in (19) and $c_{t}:=\mathrm{E}_{\hat{\mathrm{P}}}\left[\exp (\hat{\boldsymbol{B}} / \delta) \mid \hat{\mathscr{H}}_{t}\right]$ as in (17). In (40) $I$ denotes the $(n \times n)$-identity matrix. As in the proof of Proposition 1 , the key point is that the integrand in (40) with $\delta:=\bar{\delta}_{t}$ is nonnegative for every $\hat{\boldsymbol{\pi}} \in \hat{\mathcal{A}}_{t}$. To see this, we must prove that $\bar{\delta}_{t}\left(\boldsymbol{I}-\boldsymbol{R}_{y} \boldsymbol{R}_{y}^{\top}\right)-\boldsymbol{I}$ is positive semidefinite or, equivalently, that all its eigenvalues are nonnegative. But if $\alpha$ is such an eigenvalue then $1-(\alpha+1) / \bar{\delta}_{t}$ is an eigenvalue of $\boldsymbol{R}_{y} \boldsymbol{R}_{y}^{\top}$; this implies that $1-(\alpha+1) / \bar{\delta}_{t} \leq 1-1 / \bar{\delta}_{t}$ by (38) and, hence, $\alpha \geq 0$.

For the analogue of Proposition 2, we define, for $t \leq s \leq T$,

$$
\hat{\boldsymbol{\pi}}_{s}^{\star}:=\underline{\delta}_{t} \boldsymbol{R}_{s}^{\top} \zeta_{s}+\sqrt{\underline{\delta}_{t} \boldsymbol{\zeta}_{s}^{\top}\left(\boldsymbol{I}-\underline{\delta}_{t}\left(\boldsymbol{I}-\boldsymbol{R}_{s} \boldsymbol{R}_{s}^{\top}\right)\right) \zeta_{s}}(1,0, \ldots, 0)^{\top}
$$

where $\zeta$ is now determined as in (17) and (18) with $\delta:=\underline{\delta}_{t}$ and $(1,0, \ldots, 0) \in \mathbb{R}^{m}$. Using (38) and a similar reasoning as above, we see that the expression under the square root in (41) is nonnegative, and (40) simplifies to

$$
Z_{s}^{\left(\hat{\pi}^{\star}\right)}=c_{t}^{\underline{\delta}_{t}} M_{t, s}^{\left(\hat{\pi}^{\star}\right)}, \quad t \leq s \leq T,
$$

for $\hat{\pi}=\hat{\pi}^{\star}$ and $\delta=\underline{\delta}_{t}$ as in (24). As in the proof of Proposition 2, we can show that $M^{\left(\hat{\pi}^{\star}\right)}$ is a $(\mathbb{G}, \hat{\mathrm{P}})$-martingale and that $\hat{\boldsymbol{\pi}}^{\star} \in \hat{\mathcal{A}}_{t}$.

Finally, (39) is proved from the analogues of Propositions 1 and 2 similarly as in the twodimensional case. This concludes the proof outline. 
Using Theorem 4, we can of course also obtain results like Theorems 2 and 3 in the multidimensional case. We refrain from giving details because the procedure goes essentially along the same lines as in Section 4. However, we emphasize that it is important to assume that the rank of the volatility matrix $\sigma$ equals the dimension $m$ of $\boldsymbol{W}$. (In particular, we typically want at least $m$ risky assets.) This condition, implied by the assumption that $\sigma$ is invertible, is required to show that the sets $\hat{\mathscr{A}}_{t}$ and $\mathcal{A}_{t}$ fit together; compare (ii) in the proofs of Theorems 2 and 3.

\section{Acknowledgements}

It is a pleasure to thank Vicky Henderson for an enlightening discussion on the monotonicity issues in Section 5, and an anonymous referee for insisting that we improve the presentation. Financial support by the National Centre of Competence in Research 'Financial Valuation and Risk Management' (NCCR FINRISK), Project D1 (Mathematical Methods in Financial Risk Management) is gratefully acknowledged. The NCCR FINRISK is a research instrument of the Swiss National Science Foundation.

\section{References}

[1] BECHERER, D. (2006). Bounded solutions to backward SDEs with jumps for utility optimization and indifference hedging. Ann. Appl. Prob. 16, 2027-2054.

[2] Benth, F. E. AND KARLSEN, K. H. (2005). A PDE representation of the density of the minimal entropy martingale measure in stochastic volatility markets. Stochastics 77, 109-137.

[3] Delbaen, F. et al. (2002). Exponential hedging and entropic penalties. Math. Finance 12, 99-123.

[4] Dubins, L., Feldman, J., Smorodinsky, M. And Tsirelson, B. (1996). Decreasing sequences of $\sigma$-fields and a measure change for Brownian motion. Ann. Prob. 24, 882-904.

[5] Föllmer, H. ANd Schweizer, M. (1991). Hedging of contingent claims under incomplete information. In Applied Stochastic Analysis (Stoch. Monogr. 5), eds M. Davis and R. Elliott, Gordon and Breach, New York, pp. 389-414.

[6] Grasselli, M. R. and Hurd, T. R. (2007). Indifference pricing and hedging for volatility derivatives. Appl. Math. Finance 14, 303-317.

[7] Henderson, V. (2002). Valuation of claims on nontraded assets using utility maximization. Math. Finance 12, 351-373.

[8] Henderson, V. (2005). The impact of the market portfolio on the valuation, incentives and optimality of executive stock options. Quant. Finance 5, 35-47.

[9] Henderson, V. and Hobson, D. (2002). Real options with constant relative risk aversion. J. Econom. Dynam. Control 27, 329-355.

[10] Henderson, V. And Hobson, D. (2002). Substitute hedging. RISK 15, 71-75.

[11] Henderson, V. And Hobson, D. (2004). Utility indifference pricing-an overview. To appear in Indifference Pricing, ed. R. Carmona. Princeton University Press. Available at http://www.orfe.princeton.edu/ vhenders.

[12] Hu, Y., Imkeller, P. And Müller, M. (2005). Utility maximization in incomplete markets. Ann. Appl. Prob. 15, 1691-1712.

[13] Karatzas, I. And Shreve, S. (1998). Methods of Mathematical Finance. Springer, New York.

[14] KAZAMAKI, N. (1994). Continuous Exponential Martingales and BMO (Lecture Notes in Math. 1579). Springer, New York.

[15] Mania, M. And Schweizer, M. (2005). Dynamic exponential utility indifference valuation. Ann. Appl. Prob. 15, 2113-2143.

[16] Monoyios, M. (2006). Characterisation of optimal dual measures via distortion. Decis. Econom. Finance. 29, 95-119.

[17] Morlais, M.-A. (2007). Quadratic BSDEs driven by a continuous martingale and applications to the utility maximization problem. Available at http://arxiv.org/abs/math/0610749v2.

[18] Musiela, M. and Zariphopoulou, T. (2004). An example of indifference prices under exponential preferences. Finance Stoch. 8, 229-239.

[19] Schachermayer, W. (2001). Optimal investment in incomplete markets when wealth may become negative. Ann. Appl. Prob. 11, 694-734. 
[20] Stoikov, S. And Zariphopoulou, T. (2004). Optimal investments in the presence of unhedgeable risks and under CARA preferences. To appear in IMA Vol. Series.

[21] Tehranchi, M. (2004). Explicit solutions of some utility maximization problems in incomplete markets. Stochastic Process. Appl. 114, 109-125.

[22] Zariphopoulou, T. (2001). A solution approach to valuation with unhedgeable risks. Finance Stoch. 5, 61-82. 